\title{
Sc(III)催化胺对邻亚甲基苯醌氮杂迈克尔加成反应合成贝蒂碱衍生物
}

$$
\begin{aligned}
& \text { 张 硕 }{ }^{*, a} \text { 赵 宁 }{ }^{a} \text { 李庆刚 }{ }^{a} \text { 张嘉祺 }{ }^{b} \\
& \text { 侯梓桐 }^{a} \text { 刘一帆 }{ }^{a} \text { 于一涛 }{ }^{a} \text { 彭 丹*, } a \\
& \text { 王 峰 }{ }^{a} \text { 李 冰 }{ }^{a} \text { 李金辉 }{ }^{a}
\end{aligned}
$$

( ${ }^{a}$ 齐鲁工业大学(山东省科学院) 山东省科学院新材料研究所 山东省特种含硅新材料重点实验室 济南 250014) ( ${ }^{b}$ 山东省实验中学 济南 250001)

\author{
摘要 邻亚甲基苯醌化合物是一类非常活泼和重要的中间体，被广泛应用于天然产物和药物化学中. 以 2-[差基(苯基) \\ 甲基]苯酚类化合物和胺为原料, 1,2-二氯乙烷为溶剂, 在 $\mathrm{Sc}(\mathrm{III})$ 促进下原位生成邻亚甲基苯醌, 并发生氮杂迈克尔加成 \\ 反应合成贝蒂碱衍生物. 反应在封管条件下 $90{ }^{\circ} \mathrm{C}$ 搅拌 $4 \mathrm{~h}$ 完成, 以 $76 \% \sim 96 \%$ 的产率得到目标产物. \\ 关键词＼cjkstart邻亚甲基苯醌; 贝蒂碱; 氮杂迈克尔加成反应; 绿色化学
}

\section{Scandium(III)-Catalyzed Aza-Michael Addition of in Situ Generated ortho-Quinone Methides with Amines: An Efficient Access to Betti Base Derivatives}

\author{
Zhang, Shuo*,a \\ Zhao, Ning ${ }^{a}$ \\ Li, Qinggang ${ }^{a}$ \\ Zhang, Jiaqi ${ }^{b}$ \\ Hou, Zitong ${ }^{a}$ \\ Liu, Yifan ${ }^{a}$ \\ Yu, Yitao ${ }^{a}$ \\ Peng, Dan*,a \\ Wang, Feng ${ }^{a}$ \\ $\mathrm{Li}, \mathrm{Bing}^{a}$ \\ Li, Jinhui ${ }^{a}$ \\ ( ${ }^{a}$ Shandong Provincial Key Laboratory for Special Silicone-Containing Materials, Advanced Materials Institute, \\ Qilu University of Technology (Shandong Academy of Sciences), Jinan 250014) \\ ( ${ }^{b}$ Shandong Experimental High School, Jinan 250001)
}

\begin{abstract}
Quinone derivatives are not only a variety of active and important intermediate, but also widely used in the synthesis of natural products and medicinal chemistry. In the present study, the Sc(III) catalyzed aza-Michael addition to $o$-quinone methides by amines for the synthesis of Betti base derivatives was developed. The reaction was performed in a sealed tube at $90{ }^{\circ} \mathrm{C}$ for $4 \mathrm{~h}$ and the products were obtained in moderate to good yields ( $76 \% \sim 96 \%$ ).
\end{abstract}

Keywords o-quinone; Betti base; aza-Michael addtion; green chemistry

Betti 碱由 20 世纪初意大利化学家 Betti 首次合成 ${ }^{[1]}$. Betti 碱及其衍生物具有 1,3-氨基酚的结构(图 1), 是构 成具有生物活性的天然产物和药物分子的关键组分, 比 如抗菌剂 ${ }^{[2]}$ 、杀真菌剂 ${ }^{[3,4]}$ 、抗肿瘤 $[5,6]$ 以及抗结核 ${ }^{[7]}$ 等. 除 此之外, Betti 碱及其衍生物的 1,3-氨基酚配体结构能与 许多金属离子生成稳定的六元环配位结构. 作为手性配 体, Betti 碱具有的手性碳原子分别与荎环和苯环相连 接, 从而使底物在与配体中心接近时具有更好的立体选
择性. 因此 Betti 碱及其衍生物在催化不对称合成和手 性辅助合成中显示出优良的催化活性 ${ }^{[8 \sim 10]}$.

目前Betti 碱的制备方法主要是使用 Betti 反应将 醛、胺和酚三组分制备得到 Betti 碱, 该反应体系主要通 过酸性催化剂来促进反应的进行, 比如 $\mathrm{SnCl}_{4} \cdot 5 \mathrm{H}_{2} \mathrm{O}^{[11]}$ 、 聚合物负载的磺酸 ${ }^{12]}$ 、离子液体 ${ }^{[13]}$ 等, 这些合成方法有 一个或多个限制，如长反应时间长、收率低、反应条件 苛刻、使用腐蚀性或有毒试剂、价格昂贵、操作困难等.

\footnotetext{
* Corresponding authors. E-mail: e50687e@163.com; lonarpeng@aliyun.com

Received August 31, 2018; revised November 9, 2018; published online November 25, 2018.

Project supported by the Shandong Provincial Natural Science Foundation (No. ZR2017BB033), the Youth Science Funds of Shandong Academy of Sciences (No. 2018QN0030) and the National Natural Science Foundation of China (No. 51503118).

山东省自然科学基金(No. ZR2017BB033)、山东省科学院青年科学基金(No. 2018QN0030)和国家自然科学基金(No. 51503118)资助项目.
} 
因此亟需发展一种高效环保构建 Betti 碱的合成方法.<smiles>[R]c1cccc(C(c2c(O)ccc3ccccc23)N([R])[R])c1</smiles>

图 1 贝蒂碱结构通式

Figure 1 General structure of a Betti base

邻亚甲基苯醌化合物是一类非常活泼和重要的中 间体, 被广泛应用于天然产物和药物化学中 ${ }^{[14 ~ 17]}$. 因为 邻亚甲基苯醌化合物应用广泛, 其制备方法研究一直是 有机合成专家关注的热点. 现在已经有很多方法来制备 这类化合物, 例如热解、光化学反应、酸性和碱性条件 等 ${ }^{[18,19]}$, 但反应条件较为苛刻. 关于邻亚甲基苯醌的反 应类型主要分为三类: 亲核加成反应、环加成反应和 $6 \pi$ 电环化反应(Scheme 1) ${ }^{[20-32]}$. 邻亚甲基苯醌的反应研究 已经有较长的历史, 而基于 $\mathrm{P} 、 \mathrm{~S}$ 和 $\mathrm{N}$ 杂原子的迈克尔 加成反应较为成熟 ${ }^{[33 ~ 35]}$, 其中, 邻亚甲基苯醌的氮杂迈 克尔加成反应是构建含氮有机化合物的高效合成方法， 近年来有机化学家对该类反应进行了深入的研究 ${ }^{[36 ~ 45]}$, 同时, 药物化学家和生物化学家们使用该类反应发现了 较多具有生物活性的药物分子 ${ }^{[46,47]}$. 但是大部分的反应 方法仍然需要使用酸碱性催化剂. 这样的反应方法不适 用于制备含有对酸碱敏感官能团的化合物, 且在反应结 束后体系需要中和至中性, 后处理操作繁琐.

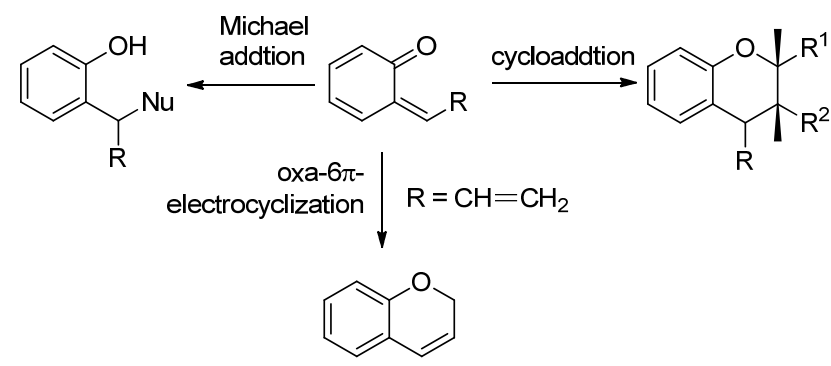

图式 1 邻亚甲基苯醌的反应类型

Scheme 1 Reaction type of $o$-quinone

基于以上的研究背景和本课题组 ${ }^{[48]}$ 在邻亚甲基苯 醌迈克尔加成领域的研究, 我们希望发展绿色环保简单 高效的胺对邻亚甲基苯醌的氮杂迈克尔加成反应的方 法. 本工作以 2-(羟基(苯基)甲基)苯酚类化合物和胺为 原料, 1,2-二氯乙烷为溶剂, 在 $\mathrm{Sc}(\mathrm{III})$ 促进下原位生成邻 亚甲基苯醌, 并发生氮杂迈克尔加成反应合成贝蒂碱衍 生物(Eq. 1). 反应在封管条件下 $90{ }^{\circ} \mathrm{C}$ 搅拌 $4 \mathrm{~h}$ 完成, 以 $76 \% \sim 96 \%$ 的产率得到目标产物. 产物的结构经 ${ }^{1} \mathrm{H}$ $\mathrm{NMR} 、{ }^{13} \mathrm{C} \mathrm{NMR}$ 和高分辨质谱确证.

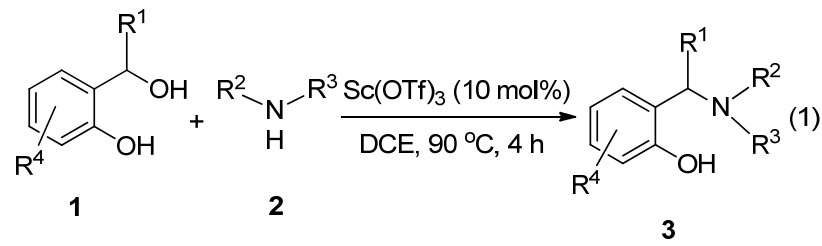

\section{1 结果与讨论}

\section{1 反应条件的优化}

首先以 2-(羟基(苯基)甲基)苯酚(1a)和硫代吗啉(2a) 为原料, 当以 $1,2-$ 二氯乙烷烷 (DCE) 为溶剂时, 分别以 $\mathrm{Ga}(\mathrm{OTf})_{3}, \mathrm{In}(\mathrm{OTf})_{3}, \mathrm{Y}(\mathrm{OTf})_{3}, \mathrm{Yb}(\mathrm{OTf})_{3}, \mathrm{AlCl}_{3}$ 和 $\mathrm{CuI}$ 为催 化剂，在 $80{ }^{\circ} \mathrm{C}$ 下反应 $6 \mathrm{~h}$ 后均没有获得目标产物 (Entries $1 \sim 6$, 表 1). 当催化剂为 $\operatorname{Sc}(\mathrm{OTf})_{3}$ 时, 可获得 75\%收率的产物(Entry 7, 表 1). 然而, 当以 $\mathrm{Sc}(\mathrm{OTf})_{3}$ 为 催化剂，分别以甲苯(Toluene)、 $N, N$-二甲基甲酰胺 (DMF)和 1,4-二氧六环 (1,4-Dioxane)为溶剂时, 在同样 的反应条件下, 产物收率都有所降低(Entries $8 \sim 10$, 表 1). 之后，对反应温度进行了考察，使用封管反应，当 升高反应温度至 $90{ }^{\circ} \mathrm{C}$, 收率上升至 $92 \%$ (Entry 11, 表 1). 但是当温度再次升高到 $100{ }^{\circ} \mathrm{C}$ 时，收率下降到 $82 \%$

表 1 反应条件优化 ${ }^{a}$

Table 1 Optimization of reaction conditions

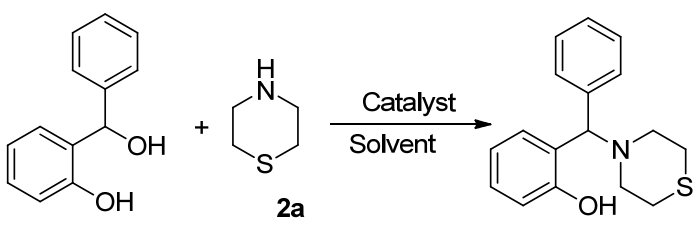

1a

3a

\begin{tabular}{cllccc}
\hline Entry & Catalyst & Solvent & Temperature $/{ }^{\circ} \mathrm{C}$ & Time $/ \mathrm{h}$ & Yield $^{b} \%$ \\
\hline 1 & $\mathrm{Ga}(\mathrm{OTf})_{3}$ & DCE & 80 & 8 & 0 \\
2 & $\mathrm{In}(\mathrm{OTf})_{3}$ & DCE & 80 & 8 & 0 \\
3 & $\mathrm{Y}(\mathrm{OTf})_{3}$ & DCE & 80 & 8 & 0 \\
4 & $\mathrm{Yb}(\mathrm{OTf})_{3}$ & DCE & 80 & 8 & 0 \\
5 & $\mathrm{AlCl}_{3}$ & DCE & 80 & 8 & 0 \\
6 & $\mathrm{CuI}$ & DCE & 80 & 8 & 0 \\
7 & $\mathrm{Sc}(\mathrm{OTf})_{3}$ & DCE & 80 & 8 & 75 \\
8 & $\mathrm{Sc}(\mathrm{OTf})_{3}$ & Toluene & 80 & 8 & 65 \\
9 & $\mathrm{Sc}(\mathrm{OTf})_{3}$ & DMF & 80 & 8 & 21 \\
10 & $\mathrm{Sc}(\mathrm{OTf})_{3}$ & $1,4-\mathrm{Dioxane}$ & 80 & 8 & 62 \\
11 & $\mathrm{Sc}(\mathrm{OTf})_{3}$ & DCE & 90 & 8 & 92 \\
12 & $\mathrm{Sc}(\mathrm{OTf})_{3}$ & DCE & 100 & 8 & 82 \\
13 & $\mathrm{Sc}(\mathrm{OTf})_{3}$ & DCE & 90 & 4 & 92 \\
14 & $\mathrm{Sc}(\mathrm{OTf})_{3}$ & DCE & 90 & 3 & 78 \\
$15^{c}$ & $\mathrm{Sc}(\mathrm{OTf})_{3}$ & DCE & 90 & 2 & 80 \\
$16^{d}$ & $\mathrm{Sc}(\mathrm{OTf})_{3}$ & DCE & 90 & 2 & 93 \\
\hline
\end{tabular}

${ }^{a}$ Reaction conditions: 2-(hydroxy(phenyl)methyl)phenol (1a, $\left.2.0 \mathrm{mmol}\right)$, thiomorpholin $(2 \mathbf{a}, 2.4 \mathrm{mmol})$, solvent $(1 \mathrm{~mL})$, run with $10 \mathrm{~mol} \% \mathrm{Sc}(\mathrm{OTf})_{3}$; ${ }^{b}$ isolated yield; ${ }^{c}$ run with $5 \mathrm{~mol} \% \mathrm{Sc}(\mathrm{OTf})_{3} ;{ }^{d}$ run with $20 \mathrm{~mol} \% \mathrm{Sc}(\mathrm{OTf})_{3}$. 
(Entry 12, 表 1). 然后，考察了反应时间，在 $90{ }^{\circ} \mathrm{C}$ 反应 时, 将反应时间缩短到 $4 \mathrm{~h}$, 产物的收率没有变化 (92\%,Entry 13，表 1)，但是当反应时间缩短至 $3 \mathrm{~h}$, 产物 的收率下降至 78\% (Entry 14, 表 1). 接着, 对催化剂用 量进行了考察. 当催化剂用量降至 $5 \mathrm{~mol} \%$ 时, 产率只有 $80 \%$ (Entry 15, 表 1). 当提升催化剂用量至 $20 \mathrm{~mol} \%$ 时, 产率变化不大 $(93 \%$, Entry 16, 表 1). 因此, 该反应的最 佳反应条件是以 $10 \mathrm{~mol} \% \mathrm{Sc}(\mathrm{OTf})_{3}$ 为催化剂、1,2-二氯 乙烷(DCE)为溶剂、 $90{ }^{\circ} \mathrm{C} 反$ 反应 $4 \mathrm{~h}$.

\section{2 底物的扩展}

在获得最佳反应条件后, 本工作将该催化体系应用 到 2-(羟基(苯基)甲基)苯酚类化合物 $\mathbf{1}$ 与仲胺 $\mathbf{2}$ 的反应 中, 来考察该催化体系的底物适用性(表 2). 从表 2 可以 看出, 对于 2-(羟基(苯基)甲基)苯酚类化合物, 取代基为 给电子的烷基、烷氧基或者为吸电子的卤素等都可以顺 利地发生氮杂迈克尔加成反应得到相应的产物, 该反应 对不同取代基的兼容性较好. 芳环上不同位置的取代基 电子效应对反应的产率有一定的影响: 当苯环上的不同
位置含甲基、叔丁基、甲氧基和乙氧基等供电子取代基 时，可获得优秀收率的产物( $90 \% \sim 96 \%$ ，表 2). 当取代 基是吸电子氯时, 收率下降, 可以得到中等收率的产物 (84\% 88\%), 而当取代基为吸电子效应更强的氟时， 产物的收率进一步的下降但仍能以中等的收率得到相 应的产物(78\% 84\%). 对于不同的仲胺，比如硫代吗 啉、吗啉、3-(4-甲氧基苯基)吗啉、4-(4-哌啶基)吗啉、 $N$-甲基-1-苯基甲胺、 $N$-甲基-1-丁胺，都可以很好地与原 位生成的邻亚甲基苯醌发生氮杂迈克尔加成反应，收率 从中等到优秀不等 $(78 \% \sim 92 \%$, 表 2).

为了进一步考察 $\mathrm{Sc}(\mathrm{OTf})_{3}$ 催化发生氮杂迈克尔加 成反应的底物适用性，将该催化体系应用到各种芐胺 $\mathbf{4}$ 的合成中(表 3). 对于 2-[羟基(苯基)甲基]苯酚类化合物, 当苯环上的不同位置含甲基、叔丁基、甲氧基和乙氧基 等供电子取代基时，可获得优秀收率的产物(90\% $95 \%$ ，表 3). 当取代基是吸电子氯时，收率下降，可以得 到中等收率的产物( $82 \% \sim 88 \%)$; 而当取代基为吸电子 效应更强的氟时, 产物的收率进一步的下降, 但仍能以

表 2 底物扩展 ${ }^{a}$

Table 2 Exploration of substrate scope<smiles>Oc1ccccc1C(c1ccccc1)N1CCSCC1</smiles>

3a, $92 \%$<smiles>Cc1cccc(C(c2ccccc2O)N2CCSCC2)c1</smiles>

3b, $94 \%$<smiles>Cc1ccccc1C(c1ccccc1O)N1CCSCC1</smiles>

3c, $92 \%$<smiles>Cc1ccc(C(c2ccccc2O)N2CCSCC2)cc1</smiles>

3d, $95 \%$<smiles>Cc1ccc(O)c(C(c2ccccc2)N2CCSCC2)c1</smiles>

3e, $94 \%$<smiles>COc1ccc(O)c(C(c2ccccc2)N2CCSCC2)c1</smiles>

3f, $93 \%$

$3 g, 96 \%$<smiles>CCOc1cccc(C(c2ccccc2)N2CCSCC2)c1O</smiles>

3h, $95 \%$

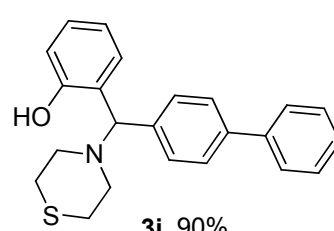

3i, $90 \%$<smiles>Oc1ccc(Cl)cc1C(c1ccccc1)N1CCSCC1</smiles>

3j, $88 \%$<smiles>Oc1ccccc1C(c1ccc(Cl)cc1)N1CCSCC1</smiles>

3k, $84 \%$<smiles>Oc1ccccc1C(c1cccc(F)c1)N1CCSCC1</smiles>

3I, $82 \%$<smiles>Oc1ccccc1C(c1ccc(F)cc1)N1CCSCC1</smiles>

3m, $78 \%$<smiles>Oc1ccccc1C(c1ccccc1)N1CCOCC1</smiles>

3n, $88 \%$<smiles>CC(C)(C)O</smiles><smiles>Cc1ccc(C(c2ccccc2O)N2CCOCC2)cc1</smiles>

3o, $90 \%$<smiles>COc1ccc(O)c(C(c2ccccc2)N2CCOCC2)c1</smiles>

3p, $92 \%$<smiles>Oc1ccccc1C(c1cccc(F)c1)N1CCOCC1</smiles>

3q, $87 \%$<smiles>COc1ccc(C2COCCN2C(c2ccccc2)c2ccccc2O)cc1</smiles>

$3 r, 92 \%$ 3s, $90 \%$<smiles>CN(Cc1ccccc1)C(c1ccccc1)c1ccccc1O</smiles><smiles>CCCCN(C)C(c1ccccc1)c1ccccc1O</smiles><smiles>Oc1ccc(F)cc1C(c1ccccc1)N1CCSCC1</smiles>

${ }^{a}$ Reaction conditions: $o$-hydroxybenzyl alcohols $1(2.0 \mathrm{mmol})$, secondary amines $2(2.4 \mathrm{mmol})$, DCE $(1 \mathrm{~mL})$, performed in a sealed tube at $90{ }^{\circ} \mathrm{C}, 4 \mathrm{~h}$. Isolated yield. 
表 31 与各种苄胺的反应 ${ }^{a}$

Table 3 Reaction of 1 with various benzylamine<smiles>Oc1ccccc1C(NCc1ccccc1)c1ccccc1</smiles>

5a, $90 \%$<smiles>Cc1cccc(CNC(c2ccccc2)c2ccccc2O)c1</smiles>

5f, $90 \%$<smiles>Oc1ccccc1C(NCc1ccccc1)c1ccc(-c2ccccc2)cc1</smiles>

$\mathbf{5 k}, 88 \%$<smiles>Cc1ccccc1CNC(c1ccccc1)c1ccccc1O</smiles>

5b, $92 \%$<smiles>Cc1ccc(O)c(C(NCc2ccccc2)c2ccccc2)c1</smiles>

5g, $90 \%$<smiles>Cc1cccc(C(NCc2ccccc2)c2ccccc2O)c1</smiles>

5c, $93 \%$<smiles>Cc1ccc(C(NCc2ccccc2)c2ccccc2O)cc1</smiles>

5h, $95 \%$<smiles>Cc1ccccc1C(NCc1ccccc1)c1ccccc1O</smiles>

5d, $92 \%$<smiles>CC(C)(C)c1cccc(C(NCc2ccccc2)c2ccccc2)c1O</smiles><smiles>Oc1ccccc1C(NCc1c(Cl)cccc1Cl)c1ccccc1</smiles>

5n, $82 \%$<smiles>Oc1ccccc1C(NCc1cccs1)c1ccccc1</smiles>

5s, $93 \%$<smiles>Cc1ccc(CNC(c2ccccc2)c2ccccc2O)cc1</smiles>

5e, $94 \%$<smiles>Oc1ccccc1C(NCc1ccc(Br)cc1)c1ccccc1</smiles>

5p, $80 \%$<smiles>Oc1ccccc1C(NCc1ccc(F)cc1)c1ccccc1</smiles>

5q, $76 \%$<smiles>Oc1ccccc1C(NCc1ccccc1)C1CCCCC1</smiles><smiles>CCOc1cccc(C(NCc2ccccc2)c2ccccc2)c1O</smiles>

5j, $89 \%$<smiles>Oc1ccccc1C(NCc1ccc(I)cc1)c1ccccc1</smiles>

5o, $82 \%$<smiles>Oc1cccc(F)c1C(NCc1ccccc1)c1ccccc1</smiles>

$5 u, 81 \%$

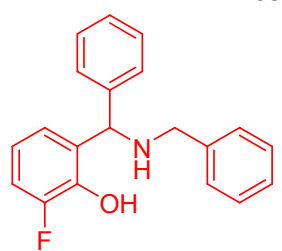

$5 v, 82 \%$

${ }^{a}$ Reaction conditions: $o$-hydroxybenzyl alcohols $1(2.0 \mathrm{mmol})$, benzylamines $2(2.4 \mathrm{mmol}), \mathrm{DCE}(1 \mathrm{~mL})$, performed in a sealed tube at $90{ }^{\circ} \mathrm{C}, 4 \mathrm{~h}$. Isolated yield.

中等的收率得到相应的产物(76\% $82 \%)$. 对于不同的 芐胺, 比如 2,6-二氯芐胺、邻甲基芐胺、间氯芐胺、间 甲基芐胺、对溴苄胺、对氟苄胺、对碘苄胺、对甲基茮 胺和 2-噻吩甲胺, 都可以很好地发生氮杂迈克尔加成反 应(表 3), 当苠胺带有给电子基团, 比如甲基, 与带有吸 电子取代基 $(\mathrm{F} 、 \mathrm{Cl} 、 \mathrm{Br}$ 和 $\mathrm{I})$ 的茮胺相比, 收率较高(表 3). 实验表明, 不同的苄胺都可以很好地与原位生成的 邻亚甲基苯醌发生氮杂迈克尔加成反应.

\section{3 反应机理研究}

基于以上的实验结果, 我们提出了一个可能的反应 机理(Scheme 2): 首先由 $\mathrm{Sc}(\mathrm{OTf})_{3}$ 催化 2-(羟基(苯基)甲 基)苯酚类化合物 1 生成邻亚甲基苯醌中间体，而后仲 胺或者苄胺对邻亚甲基苯醌中间体进行氮杂迈克尔加 成反应即得到了最终产物 $\mathbf{3}$ 或 $\mathbf{5}$.

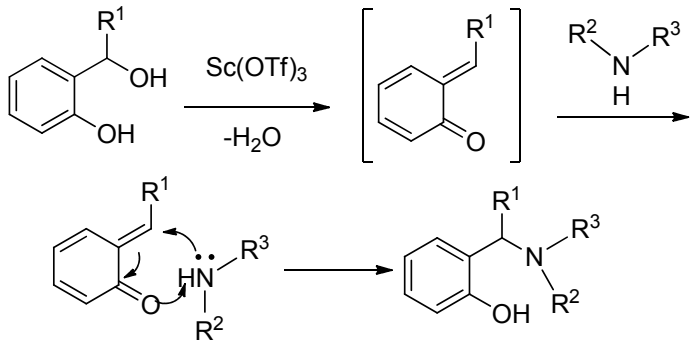

图式 2 可能的反应机理

Scheme 2 Proposed reaction mechanism

\section{2 结论}

报道了以 2-(羟基(苯基)甲基)苯酚类化合物和胺为 原料, 1,2-二氯乙烷为溶剂, 在 Sc(III)促进下原位生成邻 亚甲基苯醌，并发生氮杂迈克尔加成反应合成贝蒂碱衍 
生物. 在该催化体系下, 不同的2-(羟基(苯基)甲基)苯酚 类化合物与各种仲胺和芐胺都可顺利反应, 收率最高可 达 $96 \%$. 该体系具有催化剂廉价易得、反应条件较为温 和、底物适应性良好等优点.

\section{3 实验部分}

\section{1 仪器与试剂}

Bruker AV400 型核磁共振仪, $\mathrm{CDCl}_{3}$ 或 DMSO- $d_{6}$ 为 溶剂, TMS 为内标; Agilent Q-TOF 6510 型高分辨质谱 仪, 电喷雾离子化技术(ESI), 直接进样法进样. 柱层析 使用 $200 \sim 300$ 目硅胶.

\section{2 实验方法}

在 $38 \mathrm{~mL}$ 厚壁耐压瓶中加入 2-[差基(苯基)甲基]苯 酚类化合物 $\mathbf{1}(0.2 \mathrm{mmol}) 、$ 胺类化合物 $(0.24 \mathrm{mmol}) 、 1,2-$ 二氯乙烷 $(2.0 \mathrm{~mL})$ 和 $\mathrm{Sc}(\mathrm{OTf})_{3}(9.8 \mathrm{mg}, 10 \mathrm{~mol} \%)$, 然后 在 $90{ }^{\circ} \mathrm{C}$ 温度下封管摚拌反应 $4 \mathrm{~h}$. 反应停止后, 自然降 温至室温, 而后向反应液中加入 $6 \mathrm{~mL}$ 水, 再用二氯甲 烷 $(6 \mathrm{~mL} \times 3)$ 萃取, 有机相用无水 $\mathrm{Na}_{2} \mathrm{SO}_{4}$ 干燥, 过滤后, 所得滤液旋蒸除去溶剂得到粗产品. 粗产品经柱层析提 纯得到目标化合物. 洗脱剂为石油醚和乙酸乙酯 $(V$ : $V=40: 1)$. 薄层色谱(TLC)检测洗脱进程, 合并收集液 并旋蒸除去洗脱剂得到相应的纯品, 计算收率. 所有目 标化合物的结构经核磁共振氢谱、碳谱以及高分辨质谱 表征.

2-[苯基(硫代吗啉)甲基]苯酚(3a): 白色固体, 产率 92\%. m.p. 85.2 87.4 ${ }^{\circ} \mathrm{C} ;{ }^{1} \mathrm{H}$ NMR (400 MHz, $\left.\mathrm{CDCl}_{3}\right) \delta$ : $11.72(\mathrm{~s}, 1 \mathrm{H}), 7.36(\mathrm{~d}, J=7.1 \mathrm{~Hz}, 2 \mathrm{H}), 7.33 \sim 7.22(\mathrm{~m}$, $3 \mathrm{H}), 7.12 \sim 7.08(\mathrm{~m}, 1 \mathrm{H}), 6.86(\mathrm{dd}, J=10.9,4.2 \mathrm{~Hz}, 2 \mathrm{H})$, $6.68(\mathrm{td}, J=7.5,0.9 \mathrm{~Hz}, 1 \mathrm{H}), 4.56(\mathrm{~s}, 1 \mathrm{H}), 2.82 \sim 2.73(\mathrm{~m}$, $2 \mathrm{H}), 2.70 \sim 2.64(\mathrm{~m}, 6 \mathrm{H}) ;{ }^{13} \mathrm{C} \mathrm{NMR}\left(\mathrm{CDCl}_{3}, 100 \mathrm{MHz}\right) \delta$ : $156.69,138.37,129.49,128.95,128.93,128.75,128.21$, 124.72, 119.45, 117.05, 76.34, 53.23, 28.13; HRMS (ESI) calcd for $\mathrm{C}_{17} \mathrm{H}_{20} \mathrm{NOS}[\mathrm{M}+\mathrm{H}]^{+}$286.1260, found 286.1261 .

2-[硫代吗啉(间甲苯基)甲基]苯酚(3b)：白色固体， 产率 94\%. m.p. $65.5 \sim 68.2{ }^{\circ} \mathrm{C}^{1} \mathrm{H}$ NMR $(400 \mathrm{MHz}$, $\left.\mathrm{CDCl}_{3}\right) \delta: 11.75(\mathrm{~s}, 1 \mathrm{H}), 7.24 \sim 7.16(\mathrm{~m}, 3 \mathrm{H}), 7.13 \sim 7.07$ $(\mathrm{m}, 2 \mathrm{H}), 6.89 \sim 6.84(\mathrm{~m}, 2 \mathrm{H}), 6.71 \sim 6.67(\mathrm{~m}, 1 \mathrm{H}), 4.52$ (s, 1H), 2.83 2.67 (m, 8H), $2.32(\mathrm{~s}, 3 \mathrm{H}) ;{ }^{13} \mathrm{C} \mathrm{NMR}$ $\left(\mathrm{CDCl}_{3}, 100 \mathrm{MHz}\right) \delta$ : $156.67,138.57,138.30,129.48$, 129.43, 128.96, 128.80, 128.64, 125.94, 124.78, 119.39, $116.99,76.40,53.24,28.13,21.58$; HRMS (ESI) calcd for $\mathrm{C}_{18} \mathrm{H}_{22} \mathrm{NOS}[\mathrm{M}+\mathrm{H}]^{+}$300.1417, found 300.1417.

2-[硫代吗啉(邻甲苯基)甲基]苯酚(3c)：白色固体, 产率 92\%. m.p. $70.3 \sim 71.4{ }^{\circ} \mathrm{C} ;{ }^{1} \mathrm{H}$ NMR $(400 \mathrm{MHz}$,
$\left.\mathrm{CDCl}_{3}\right) \delta: 11.84(\mathrm{~s}, 1 \mathrm{H}), 7.51(\mathrm{dd}, J=5.6,3.2 \mathrm{~Hz}, 1 \mathrm{H})$, $7.23 \sim 7.08(\mathrm{~m}, 4 \mathrm{H}), 6.86 \sim 6.82(\mathrm{~m}, 2 \mathrm{H}), 6.68$ (td, $J=7.5$, $1.0 \mathrm{~Hz}, 1 \mathrm{H}), 4.98(\mathrm{~s}, 1 \mathrm{H}), 2.90 \sim 2.70(\mathrm{~m}, 8 \mathrm{H}), 2.47(\mathrm{~s}$, $3 \mathrm{H}) ;{ }^{13} \mathrm{C}$ NMR $\left(\mathrm{CDCl}_{3}, 100 \mathrm{MHz}\right) \delta: 156.76,137.43$, $135.89,130.94,129.16,128.57,128.53,127.75,127.10$, $125.37,119.47,117.17,70.08,52.95,28.33,20.84$; HRMS (ESI) calcd for $\mathrm{C}_{18} \mathrm{H}_{22} \mathrm{NOS}[\mathrm{M}+\mathrm{H}]^{+}$300.1417, found 300.1415 .

2-[硫代吗啉(对甲苯基)甲基]苯酚(3d)：白色固体, 产率 95\%. m.p. 72.6 74.5 ${ }^{\circ} \mathrm{C}$; ${ }^{1} \mathrm{H}$ NMR $(400 \mathrm{MHz}$, $\left.\mathrm{CDCl}_{3}\right) \delta: 11.76(\mathrm{~s}, 1 \mathrm{H}), 7.24(\mathrm{~d}, J=7.9 \mathrm{~Hz}, 2 \mathrm{H}), 7.12 \sim$ $7.07(\mathrm{~m}, 3 \mathrm{H}), 6.85(\mathrm{dd}, J=11.0,4.4 \mathrm{~Hz}, 2 \mathrm{H}), 6.67(\mathrm{td}$, $J=7.5,1.0 \mathrm{~Hz}, 1 \mathrm{H}), 4.53(\mathrm{~s}, 1 \mathrm{H}), 2.81 \sim 2.63(\mathrm{~m}, 8 \mathrm{H})$, $2.29(\mathrm{~s}, 3 \mathrm{H}) ;{ }^{13} \mathrm{C} \mathrm{NMR}\left(\mathrm{CDCl}_{3}, 100 \mathrm{MHz}\right) \delta: 156.75$, 137.94, 135.29, 129.62, 129.46, 128.88, 128.66, 124.90, 119.42, 117.00, 76.01, 53.17, 28.15, 21.16; HRMS (ESI) calcd for $\mathrm{C}_{18} \mathrm{H}_{22} \mathrm{NOS}[\mathrm{M}+\mathrm{H}]^{+}$300.1417, found 300.1416 .

4-甲基-2-[苯基(硫代吗啉)甲基]苯酚(3e)：白色固 体, 产率 94\%. m.p. 54.3 56.1 ${ }^{\circ} \mathrm{C} ;{ }^{1} \mathrm{H}$ NMR $(400 \mathrm{MHz}$, $\left.\mathrm{CDCl}_{3}\right) \delta: 11.43(\mathrm{~s}, 1 \mathrm{H}), 7.37(\mathrm{~d}, J=7.2 \mathrm{~Hz}, 2 \mathrm{H}), 7.26$ (ddd, $J=10.8,9.7,5.4 \mathrm{~Hz}, 3 \mathrm{H}), 6.90(\mathrm{dd}, J=8.2,1.6 \mathrm{~Hz}$, $1 \mathrm{H}), 6.75(\mathrm{~d}, J=8.2 \mathrm{~Hz}, 1 \mathrm{H}), 6.69(\mathrm{~s}, 1 \mathrm{H}), 4.46(\mathrm{~s}, 1 \mathrm{H})$, $2.82 \sim 2.63(\mathrm{~m}, 8 \mathrm{H}), 2.12(\mathrm{~s}, 3 \mathrm{H}) ;{ }^{13} \mathrm{C} \mathrm{NMR}\left(\mathrm{CDCl}_{3}, 100\right.$ MHz) $\delta: 154.11,138.92,129.91,129.26,128.99,128.83$, $128.49,128.15,124.53,116.86,76.54,53.40,28.14,20.56$; HRMS (ESI) calcd for $\mathrm{C}_{18} \mathrm{H}_{22} \mathrm{NOS}[\mathrm{M}+\mathrm{H}]^{+} 300.1417$, found 300.1417 .

2-叔丁基-6-[苯基(硫代吗啉)甲基]苯酚(3f): 无色油 状液体, 产率 93\%. ${ }^{1} \mathrm{H}$ NMR (400 MHz, $\left.\mathrm{CDCl}_{3}\right) \delta: 12.14$ (s, 1H), 7.39 (d, $J=6.9 \mathrm{~Hz}, 2 \mathrm{H}), 7.34 \sim 7.25(\mathrm{~m}, 3 \mathrm{H}), 7.15$ (dd, $J=7.7,1.4 \mathrm{~Hz}, 1 \mathrm{H}), 6.75(\mathrm{~d}, J=7.1 \mathrm{~Hz}, 1 \mathrm{H}), 6.64(\mathrm{t}$, $J=7.6 \mathrm{~Hz}, 1 \mathrm{H}), 4.59$ (s, 1H), $2.80(\mathrm{~d}, J=53.0 \mathrm{~Hz}, 8 \mathrm{H})$, $1.44(\mathrm{~s}, 9 \mathrm{H}) ;{ }^{13} \mathrm{C}$ NMR $\left(\mathrm{CDCl}_{3}, 100 \mathrm{MHz}\right) \delta: 155.86$, $138.39,137.27,129.00,128.85,128.07,127.50,125.84$, 124.56, 118.44, 76.72, 52.95, 34.84, 29.47, 27.97; HRMS (ESI) calcd for $\mathrm{C}_{21} \mathrm{H}_{28} \mathrm{NOS}[\mathrm{M}+\mathrm{H}]^{+} 342.1886$, found 342.1885 .

4-甲氧基-2-[苯基(硫代吗啉)甲基]苯酚(3g)：白色固 体, 产率 96\%. m.p. 113.2 115.5 ${ }^{\circ} \mathrm{C} ;{ }^{1} \mathrm{H}$ NMR (400 $\left.\mathrm{MHz}, \mathrm{CDCl}_{3}\right) \delta: 11.21(\mathrm{~s}, 1 \mathrm{H}), 7.38(\mathrm{~d}, J=7.1 \mathrm{~Hz}, 2 \mathrm{H})$, $7.32 \sim 7.24(\mathrm{~m}, 3 \mathrm{H}), 6.79(\mathrm{~d}, J=8.8 \mathrm{~Hz}, 1 \mathrm{H}), 6.68$ (dd, $J=$ 8.8, $3.0 \mathrm{~Hz}, 1 \mathrm{H}), 6.47(\mathrm{~d}, J=2.9 \mathrm{~Hz}, 1 \mathrm{H}), 4.49$ (s, 1H), $3.63(\mathrm{~s}, 3 \mathrm{H}), 2.83 \sim 2.65(\mathrm{~m}, 8 \mathrm{H}) ;{ }^{13} \mathrm{C}$ NMR $\left(\mathrm{CDCl}_{3}, 100\right.$ MHz) $\delta: 152.60,150.28,138.41,128.96,128.86,128.22$, 
125.39, 117.42, 115.23, 113.65, 76.43, 55.64, 53.30, 28.10; HRMS (ESI) calcd for $\mathrm{C}_{18} \mathrm{H}_{22} \mathrm{NO}_{2} \mathrm{~S}[\mathrm{M}+\mathrm{H}]^{+} 316.1366$, found 316.1366 .

2-乙氧基-6-[苯基(硫代吗啉)甲基]苯酚(3h): 白色 固体, 产率 95\%. m.p. 117.1 119.2 ${ }^{\circ} \mathrm{C} ;{ }^{1} \mathrm{H}$ NMR (400 $\left.\mathrm{MHz}, \mathrm{CDCl}_{3}\right) \delta: 11.75$ (s, 1H), 7.39 (d, $\left.J=7.1 \mathrm{~Hz}, 2 \mathrm{H}\right)$, $7.28(\mathrm{dt}, J=6.9,4.5 \mathrm{~Hz}, 3 \mathrm{H}), 6.75(\mathrm{dd}, J=8.0,1.0 \mathrm{~Hz}$, $1 \mathrm{H}), 6.64$ (t, $J=7.9 \mathrm{~Hz}, 1 \mathrm{H}), 6.53$ (d, $J=7.6 \mathrm{~Hz}, 1 \mathrm{H}), 4.57$ $(\mathrm{s}, 1 \mathrm{H}), 4.08(\mathrm{q}, J=7.0 \mathrm{~Hz}, 2 \mathrm{H}), 2.86 \sim 2.68(\mathrm{~m}, 8 \mathrm{H}), 1.49$ $(\mathrm{t}, J=7.0 \mathrm{~Hz}, 3 \mathrm{H}) ;{ }^{13} \mathrm{C} \mathrm{NMR}\left(\mathrm{CDCl}_{3}, 100 \mathrm{MHz}\right) \delta: 147.64$, $146.17,138.64,128.86,128.84,128.08,125.17,121.21$, 118.94, 111.96, 75.99, 64.28, 53.33, 28.10, 15.00; HRMS (ESI) calcd for $\mathrm{C}_{19} \mathrm{H}_{24} \mathrm{NO}_{2} \mathrm{~S}[\mathrm{M}+\mathrm{H}]^{+}$330.1522, found 330.1520

2-([1,1'-二苯基]-4-基(硫代吗啉)甲基)苯酚(3i): 白 色固体, 产率 90\%. m.p. 112.5 $114.2{ }^{\circ} \mathrm{C} ;{ }^{1} \mathrm{H}$ NMR (400 $\left.\mathrm{MHz}, \mathrm{CDCl}_{3}\right) \delta: 11.74(\mathrm{~s}, 1 \mathrm{H}), 7.54(\mathrm{dd}, J=8.1,2.3 \mathrm{~Hz}$, $4 \mathrm{H}), 7.52 \sim 7.40(\mathrm{~m}, 4 \mathrm{H}), 7.33(\mathrm{t}, J=7.3 \mathrm{~Hz}, 1 \mathrm{H}), 7.16 \sim$ $7.11(\mathrm{~m}, 1 \mathrm{H}), 6.93 \sim 6.92(\mathrm{~m}, 1 \mathrm{H}), 6.88(\mathrm{~d}, J=8.1 \mathrm{~Hz}, 1 \mathrm{H})$, $6.74 \sim 6.70(\mathrm{~m}, 1 \mathrm{H}), 4.61(\mathrm{~s}, 1 \mathrm{H}), 2.88 \sim 2.69(\mathrm{~m}, 8 \mathrm{H}) ;{ }^{13} \mathrm{C}$ NMR $\left(\mathrm{CDCl}_{3}, 100 \mathrm{MHz}\right) \delta: 156.67,141.07,140.43$, $137.35,129.45,129.29$, 128.84, 128.80, 127.62, 127.50, 127.04, 124.67, 119.51, 117.10, 76.04, 53.27, 28.14; HRMS (ESI) calcd for $\mathrm{C}_{23} \mathrm{H}_{24} \mathrm{NOS}[\mathrm{M}+\mathrm{H}]^{+} 362.1573$, found 362.1575 .

4-氯-2-[苯基(硫代吗啉)甲基]苯酚 $(3 \mathbf{j})$ : 白色固体, 产率 88\%. 95.3 96.1 ${ }^{\circ} \mathrm{C} ;{ }^{1} \mathrm{H}$ NMR (400 $\mathrm{MHz} \mathrm{CDCl}_{3}$ ) $\delta: 11.84(\mathrm{~s}, 1 \mathrm{H}), 7.36 \sim 7.29(\mathrm{~m}, 5 \mathrm{H}), 7.06(\mathrm{dd}, J=8.7$, $2.5 \mathrm{~Hz}, 1 \mathrm{H}), 6.86(\mathrm{~d}, J=2.5 \mathrm{~Hz}, 1 \mathrm{H}), 6.79$ (d, $J=8.7 \mathrm{~Hz}$, $1 \mathrm{H}), 4.52(\mathrm{~s}, 1 \mathrm{H}), 3.05 \sim 2.55(\mathrm{~m}, 8 \mathrm{H}) ;{ }^{13} \mathrm{C} \mathrm{NMR}\left(\mathrm{CDCl}_{3}\right.$, $100 \mathrm{MHz}) \delta: 155.41,137.56,129.09,128.84,128.60$, 128.52, 126.19, 123.87, 118.43, 118.40, 75.94, 53.18, 28.04; HRMS (ESI) calcd for $\mathrm{C}_{17} \mathrm{H}_{19} \mathrm{CINOS}[\mathrm{M}+\mathrm{H}]^{+}$ 320.0870 , found 320.0872 .

2-(4-氯苯基)(硫代吗啉)苯酚(3k)：白色固体，产率 84\%. m.p. 102.5 104.1 ${ }^{\circ} \mathrm{C} ;{ }^{1} \mathrm{H}$ NMR (400 MHz, $\mathrm{CDCl}_{3}$ ) $\delta$ : $11.57(\mathrm{~s}, 1 \mathrm{H}), 7.30(\mathrm{q}, J=8.7 \mathrm{~Hz}, 4 \mathrm{H}), 7.14 \sim 7.10(\mathrm{~m}$, $1 \mathrm{H}), 6.85(\mathrm{~d}, J=8.1 \mathrm{~Hz}, 2 \mathrm{H}), 6.70(\mathrm{td}, J=7.6,1.0 \mathrm{~Hz}$, $1 \mathrm{H}), 4.54(\mathrm{~s}, 1 \mathrm{H}), 2.82 \sim 2.69(\mathrm{~m}, 8 \mathrm{H}) ;{ }^{13} \mathrm{C} \mathrm{NMR}\left(\mathrm{CDCl}_{3}\right.$, $100 \mathrm{MHz}) \delta: 156.47,137.02,134.05,130.17,129.29$, 129.16, 128.96, 124.28, 119.61, 117.21, 75.61, 53.24, 28.07; HRMS (ESI) calcd for $\mathrm{C}_{17} \mathrm{H}_{19} \mathrm{CINOS}[\mathrm{M}+\mathrm{H}]^{+}$ 320.0870 , found 320.0870 .

2-[(3-氟苯基)(硫代吗啉)甲基]苯酚(3I)：白色固体, 产率 82\%. m.p. 115.3 $117.5{ }^{\circ} \mathrm{C}$; ${ }^{1} \mathrm{H}$ NMR (400 MHz,
$\left.\mathrm{CDCl}_{3}\right) \delta: 11.52(\mathrm{~s}, 1 \mathrm{H}), 7.26(\mathrm{dt}, J=7.8,4.8 \mathrm{~Hz}, 1 \mathrm{H})$, 7.13 (ddd, $J=8.0,7.4,4.7 \mathrm{~Hz}, 3 \mathrm{H}), 6.98 \sim 6.94(\mathrm{~m}, 1 \mathrm{H})$, $6.87(\mathrm{dd}, J=9.5,4.0 \mathrm{~Hz}, 2 \mathrm{H}), 6.71(\mathrm{td}, J=7.6,0.8 \mathrm{~Hz}$, $1 \mathrm{H}), 4.54(\mathrm{~s}, 1 \mathrm{H}), 2.83 \sim 2.67(\mathrm{~m}, 8 \mathrm{H}) ;{ }^{13} \mathrm{C} \mathrm{NMR}\left(\mathrm{CDCl}_{3}\right.$, $100 \mathrm{MHz}) \delta$ : $162.98(\mathrm{~d}, J=245.6 \mathrm{~Hz}), 156.48,141.08(\mathrm{~d}$, $J=6.7 \mathrm{~Hz}), 130.50(\mathrm{~d}, J=8.2 \mathrm{~Hz}), 129.34,128.99$, 124.46, 124.18, 119.59, 117.22, 115.63 (d, $J=21.6 \mathrm{~Hz}$ ), 115.19 (d, $J=20.9 \mathrm{~Hz}), 75.87,53.28,28.07$; HRMS (ESI) calcd for $\mathrm{C}_{17} \mathrm{H}_{19}$ FNOS $[\mathrm{M}+\mathrm{H}]^{+}$304.1166, found 304.1166 .

2-[(4-氟苯基)(硫代吗啉)甲基]苯酚(3m): 白色固体, 产率 78\%. m.p. $119.6 \sim 121.3{ }^{\circ} \mathrm{C} ;{ }^{1} \mathrm{H}$ NMR $(400 \mathrm{MHz}$, $\left.\mathrm{CDCl}_{3}\right) \delta: 11.66(\mathrm{~s}, 1 \mathrm{H}), 7.35(\mathrm{dd}, J=8.2,5.5 \mathrm{~Hz}, 2 \mathrm{H})$, $7.15 \sim 7.11(\mathrm{~m}, 1 \mathrm{H}), 7.01(\mathrm{t}, J=8.7 \mathrm{~Hz}, 2 \mathrm{H}), 6.86(\mathrm{~d}, J=$ $8.0 \mathrm{~Hz}, 2 \mathrm{H}), 6.71(\mathrm{dd}, J=11.6,4.2 \mathrm{~Hz}, 1 \mathrm{H}), 4.57$ (s, 1H), $2.84 \sim 2.73(\mathrm{~m}, 8 \mathrm{H}) ;{ }^{13} \mathrm{C} \mathrm{NMR}\left(\mathrm{CDCl}_{3}, 100 \mathrm{MHz}\right) \delta$ : 162.48 (d, $J=245.9 \mathrm{~Hz}), 156.48,134.22$ (d, $J=3.2 \mathrm{~Hz})$, $130.54(\mathrm{~d}, J=7.9 \mathrm{~Hz}), 129.32,128.87,124.53,119.59$, 117.15, 115.86, 75.49, 53.19, 28.07; HRMS (ESI) calcd for $\mathrm{C}_{17} \mathrm{H}_{19} \mathrm{FNOS}[\mathrm{M}+\mathrm{H}]^{+}$304.1166, found 304.1167.

2-[吗啉(苯基)甲基]苯酚(3n): 白色固体，产率 $88 \%$. m.p. 115.2 $116.3{ }^{\circ} \mathrm{C}\left(\right.$ lit. ${ }^{[49]}$ m.p. $\left.112 \sim 114{ }^{\circ} \mathrm{C}\right) ;{ }^{1} \mathrm{H}$ NMR $\left(400 \mathrm{MHz}, \mathrm{CDCl}_{3}\right) \delta: 11.71(\mathrm{~s}, 1 \mathrm{H}), 7.41(\mathrm{~d}, J=7.1 \mathrm{~Hz}$, $2 \mathrm{H}), 7.30 \sim 7.21(\mathrm{~m}, 3 \mathrm{H}), 7.13 \sim 7.09(\mathrm{~m}, 1 \mathrm{H}), 6.94 \sim 6.92$ (m, 1H), $6.86(\mathrm{~d}, J=8.1 \mathrm{~Hz}, 1 \mathrm{H}), 6.71(\mathrm{td}, J=7.5,0.9 \mathrm{~Hz}$, $1 \mathrm{H}), 4.39(\mathrm{~s}, 1 \mathrm{H}), 3.77 \sim 3.72(\mathrm{~m}, 4 \mathrm{H}), 2.58 \sim 2.42(\mathrm{~m}$, $4 \mathrm{H}) ;{ }^{13} \mathrm{C} \mathrm{NMR}\left(\mathrm{CDCl}_{3}, 100 \mathrm{MHz}\right) \delta: 156.15,139.33$, $129.43,128.97,128.73,128.57,128.14,124.84,119.64$, 117.08, 76.87, 66.92, 52.28; HRMS (ESI) calcd for $\mathrm{C}_{17} \mathrm{H}_{20} \mathrm{NO}_{2}[\mathrm{M}+\mathrm{H}]^{+}$270.1489, found 270.1490.

2-[吗啉(对甲苯基)甲基]苯酚(3o): 白色固体，产率 90\%. m.p. $110.3 \sim 111.2{ }^{\circ} \mathrm{C}$ (lit. ${ }^{[50]}$ m.p. $\left.110 \sim 111{ }^{\circ} \mathrm{C}\right) ;{ }^{1} \mathrm{H}$ NMR (400 MHz, $\left.\mathrm{CDCl}_{3}\right) \delta: 11.74(\mathrm{~s}, 1 \mathrm{H}), 7.29$ (d, $J=7.6$ $\mathrm{Hz}, 2 \mathrm{H}), 7.11 \sim 7.07(\mathrm{~m}, 3 \mathrm{H}), 6.92(\mathrm{dd}, J=7.6,1.2 \mathrm{~Hz}$, $1 \mathrm{H}), 6.85(\mathrm{dd}, J=8.1,0.8 \mathrm{~Hz}, 1 \mathrm{H}), 6.70(\mathrm{td}, J=7.5,1.0$ $\mathrm{Hz}, 1 \mathrm{H}), 4.36(\mathrm{~s}, 1 \mathrm{H}), 3.76 \sim 3.71(\mathrm{~m}, 4 \mathrm{H}), 2.56 \sim 2.41(\mathrm{~m}$, $4 \mathrm{H}), 2.27(\mathrm{~s}, 3 \mathrm{H}) ;{ }^{13} \mathrm{C} \mathrm{NMR}\left(\mathrm{CDCl}_{3}, 100 \mathrm{MHz}\right) \delta: 156.19$, $137.88,136.31,129.63,129.40,128.64,128.50,125.04$, 119.62, 117.03, 76.52, 66.95, 52.23, 21.11; HRMS (ESI) calcd for $\mathrm{C}_{18} \mathrm{H}_{22} \mathrm{NO}_{2}[\mathrm{M}+\mathrm{H}]^{+}$284.1645, found 284.1647.

4-甲氧基-2-[吗啉(苯基)甲基]苯酚(3p): 白色固体, 产率 92\%. m.p. 87.4 89.2 ${ }^{\circ} \mathrm{C}$ (lit. ${ }^{[51]}$ m.p. $\left.87 \sim 89{ }^{\circ} \mathrm{C}\right) ;{ }^{1} \mathrm{H}$ NMR (400 MHz, $\left.\mathrm{CDCl}_{3}\right) \delta: 11.21(\mathrm{~s}, 1 \mathrm{H}), 7.42$ (d, $J=7.1$ $\mathrm{Hz}, 2 \mathrm{H}), 7.26$ (dtd, $J=10.0,7.5,2.8 \mathrm{~Hz}, 3 \mathrm{H}), 6.79$ (d, $J=$ $8.8 \mathrm{~Hz}, 1 \mathrm{H}), 6.68(\mathrm{dd}, J=8.8,3.0 \mathrm{~Hz}, 1 \mathrm{H}), 6.51(\mathrm{~d}, J=2.9$ 
$\mathrm{Hz}, 1 \mathrm{H}), 4.32$ (s, 1H), 3.78 3.73 (m, 4H), 3.65 (s, 3H), $2.58 \sim 2.41(\mathrm{~m}, 4 \mathrm{H}) ;{ }^{13} \mathrm{C}$ NMR $\left(\mathrm{CDCl}_{3}, 100 \mathrm{MHz}\right) \delta$ : $152.72,149.80,139.27,128.96,128.56,128.17,125.41$, 117.50, 114.95, 113.80, 76.97, 66.91, 55.61, 52.32; HRMS (ESI) calcd for $\mathrm{C}_{18} \mathrm{H}_{22} \mathrm{NO}_{3}[\mathrm{M}+\mathrm{H}]^{+} 300.1594$, found 300.1595 .

2-[(3-氟苯基)(吗啉)甲基]苯酚(3q): 白色固体, 产 率 87\%. m.p. 135.2 137.1 ${ }^{\circ} \mathrm{C} ;{ }^{1} \mathrm{H}$ NMR $(400 \mathrm{MHz}$, $\left.\mathrm{CDCl}_{3}\right) \delta: 11.52(\mathrm{~s}, 1 \mathrm{H}), 7.29 \sim 7.11(\mathrm{~m}, 4 \mathrm{H}), 6.97 \sim 6.93$ $(\mathrm{m}, 2 \mathrm{H}), 6.89 \sim 6.86(\mathrm{~m}, 1 \mathrm{H}), 6.74(\mathrm{td}, J=7.5,0.9 \mathrm{~Hz}$, $1 \mathrm{H}), 4.38(\mathrm{~s}, 1 \mathrm{H}), 3.80 \sim 3.76(\mathrm{~m}, 4 \mathrm{H}), 2.60 \sim 2.43(\mathrm{~m}$, $4 \mathrm{H}) ;{ }^{13} \mathrm{C}$ NMR $\left(\mathrm{CDCl}_{3}, 100 \mathrm{MHz}\right) \delta: 163.00(\mathrm{~d}, J=245.5$ Hz), 155.97, 141.92 (d, $J=6.4 \mathrm{~Hz}), 130.50$ (d, $J=8.2 \mathrm{~Hz})$, $129.31,129.00,124.27,124.13,119.76,117.25,115.42$, $115.13(\mathrm{~d}, J=20.9 \mathrm{~Hz})$; HRMS (ESI) calcd for $\mathrm{C}_{17} \mathrm{H}_{19} \mathrm{FNO}_{2}[\mathrm{M}+\mathrm{H}]^{+}$288.1394, found 288.1394.

2-\{[3-(4-甲氧基苯基)吗啉](苯基)甲基 $\}$ 苯酚 (3r): 白色固体, 产率 92\%. m.p. 146.3 147.1 ${ }^{\circ} \mathrm{C} ;{ }^{1} \mathrm{H}$ NMR $\left(400 \mathrm{MHz}, \mathrm{CDCl}_{3}\right) \delta: 12.79(\mathrm{~s}, 1 \mathrm{H}), 7.38 \sim 7.30(\mathrm{~m}, 5 \mathrm{H})$, $7.12 \sim 6.94(\mathrm{~m}, 5 \mathrm{H}), 6.87 \sim 6.85(\mathrm{~m}, 1 \mathrm{H}), 6.58(\mathrm{dd}, J=$ 11.0, $4.0 \mathrm{~Hz}, 1 \mathrm{H}), 6.47(\mathrm{~d}, J=7.8 \mathrm{~Hz}, 1 \mathrm{H}), 5.47(\mathrm{~s}, 1 \mathrm{H})$, $3.81(\mathrm{~s}, 3 \mathrm{H}), 3.59$ (dd, $J=9.7,3.2 \mathrm{~Hz}, 1 \mathrm{H}), 3.43$ (dd, $J=$ $10.7,2.2 \mathrm{~Hz}, 1 \mathrm{H}), 3.08 \sim 3.00(\mathrm{~m}, 2 \mathrm{H}), 2.86 \sim 2.83(\mathrm{~m}$, $1 \mathrm{H}), 2.58 \sim 2.55(\mathrm{~m}, 1 \mathrm{H}), 2.50 \sim 2.48(\mathrm{~m}, 1 \mathrm{H}) ;{ }^{13} \mathrm{C} \mathrm{NMR}$ $\left(\mathrm{CDCl}_{3}, 100 \mathrm{MHz}\right) \delta: 159.35,158.65,133.26,133.00$, $131.46,129.95,129.52,128.75,128.39,128.11,122.71$, 118.56, 116.58, 114.61, 67.43, 66.11, 55.35, 49.97, 36.86, 28.05; HRMS (ESI) calcd for $\mathrm{C}_{24} \mathrm{H}_{26} \mathrm{NO}_{3}[\mathrm{M}+\mathrm{H}]^{+}$ 376.1907, found 376.1908 .

2-[吗啉(邻甲苯基)甲基] 苯酚(3s): 白色固体, 产率 90\%. m.p. 46.2 48.5 ${ }^{\circ} \mathrm{C} ;{ }^{1} \mathrm{H}$ NMR (400 MHz, $\left.\mathrm{CDCl}_{3}\right) \delta$ : $11.87(\mathrm{~s}, 1 \mathrm{H}), 7.60 \sim 7.58(\mathrm{~m}, 1 \mathrm{H}), 7.14 \sim 7.09(\mathrm{~m}, 4 \mathrm{H})$, $6.91 \sim 6.84(\mathrm{~m}, 2 \mathrm{H}), 6.69(\mathrm{td}, J=7.5,1.0 \mathrm{~Hz}, 1 \mathrm{H}), 4.87(\mathrm{~s}$, $1 \mathrm{H}), 3.76 \sim 3.71(\mathrm{~m}, 4 \mathrm{H}), 2.64 \sim 2.62(\mathrm{~m}, 2 \mathrm{H}), 2.49(\mathrm{~s}$, $3 \mathrm{H}), 2.46 \sim 2.45(\mathrm{~m}, 2 \mathrm{H}) ;{ }^{13} \mathrm{C} \mathrm{NMR}\left(\mathrm{CDCl}_{3}, 100 \mathrm{MHz}\right) \delta$ : $156.40,137.97,135.61,130.91,129.09,128.60,128.32$, $127.71,127.18,125.32,119.58,117.25,70.30,67.03$, 51.87, 20.91; HRMS (ESI) calcd for $\mathrm{C}_{18} \mathrm{H}_{22} \mathrm{NO}_{2}[\mathrm{M}+\mathrm{H}]^{+}$ 284.1645 , found 284.1648 .

2-[(4-吗啉哌啶-1-基)(苯基)甲基]苯酚(3t)：白色固 体, 产率 82\%. m.p. 83.5 85.1 ${ }^{\circ} \mathrm{C} ;{ }^{1} \mathrm{H}$ NMR $(400 \mathrm{MHz}$, $\left.\mathrm{CDCl}_{3}\right) \delta: 12.11(\mathrm{~s}, 1 \mathrm{H}), 7.39 \sim 7.25(\mathrm{~m}, 5 \mathrm{H}), 7.13 \sim 7.08$ (m, $1 \mathrm{H}), 6.90 \sim 6.84(\mathrm{~m}, 2 \mathrm{H}), 6.69(\mathrm{td}, J=7.5,0.9 \mathrm{~Hz}$, 1H), $4.46(\mathrm{~s}, 1 \mathrm{H}), 3.71 \sim 3.69(\mathrm{~m}, 4 \mathrm{H}), 3.23$ (d, $J=10.5$ $\mathrm{Hz}, 1 \mathrm{H}), 2.84 \sim 2.81(\mathrm{~m}, 1 \mathrm{H}), 2.55 \sim 2.50(\mathrm{~m}, 4 \mathrm{H}), 2.20$ (tt,
$J=11.2,3.7 \mathrm{~Hz}, 1 \mathrm{H}), 2.11 \sim 2.10(\mathrm{~m}, 1 \mathrm{H}), 1.97(\mathrm{t}, J=11.3$ $\mathrm{Hz}, 1 \mathrm{H}), 1.90 \sim 1.81(\mathrm{~m}, 2 \mathrm{H}), 1.66$ (ddd, $J=24.4,12.2,3.8$ $\mathrm{Hz}, 1 \mathrm{H}), 1.53$ (ddd, $J=15.5,12.2,3.7 \mathrm{~Hz}, 1 \mathrm{H}$ ); ${ }^{13} \mathrm{C}$ NMR $\left(\mathrm{CDCl}_{3}, 100 \mathrm{MHz}\right) \delta: 156.85,139.60,129.26,129.13$, $128.80,128.59,128.45,127.95,125.43,119.21,116.95$, $75.98,67.24,61.59,52.37,49.81,49.73,28.38,28.33$; HRMS (ESI) calcd for $\mathrm{C}_{22} \mathrm{H}_{29} \mathrm{~N}_{2} \mathrm{O}_{2}[\mathrm{M}+\mathrm{H}]^{+} 353.2224$, found 353.2224 .

2-[(芐基(甲基)胺)(苯基)甲基]苯酚(3u)：白色固体， 产率 80\%. m.p. 80.2 81.4 ${ }^{\circ} \mathrm{C}\left(\right.$ lit. ${ }^{[49]}$ m.p. $\left.80 \sim 81{ }^{\circ} \mathrm{C}\right) ;{ }^{1} \mathrm{H}$ NMR (400 MHz, $\left.\mathrm{CDCl}_{3}\right) \delta: 12.34(\mathrm{~s}, 1 \mathrm{H}), 7.47$ (d, $J=7.3$ $\mathrm{Hz}, 2 \mathrm{H}), 7.36 \sim 7.24(\mathrm{~m}, 8 \mathrm{H}), 7.15 \sim 7.11(\mathrm{~m}, 1 \mathrm{H}), 6.91(\mathrm{t}$, $J=7.4 \mathrm{~Hz}, 2 \mathrm{H}), 6.71(\mathrm{dd}, J=11.6,4.2 \mathrm{~Hz}, 1 \mathrm{H}), 4.70$ (s, $1 \mathrm{H}), 3.55(\mathrm{~s}, 2 \mathrm{H}), 2.16(\mathrm{~s}, 3 \mathrm{H}) ;{ }^{13} \mathrm{C}$ NMR $\left(\mathrm{CDCl}_{3}, 100\right.$ MHz) $\delta: 156.99,137.23,129.34,129.19,129.03,128.86$, $128.71,128.68,128.65,128.12,127.61,125.42,119.24$, $117.01,75.55,59.65,39.19$; HRMS (ESI) calcd for $\mathrm{C}_{21} \mathrm{H}_{22} \mathrm{NO}[\mathrm{M}+\mathrm{H}]^{+}$304.1696, found 304.1695.

2-[(丁基(甲基)胺)(苯基)甲基]苯酚(3v): 无色油状 液体, 产率 78\%. ${ }^{1} \mathrm{H}$ NMR $\left(400 \mathrm{MHz}, \mathrm{CDCl}_{3}\right) \delta: 12.53$ (s, $1 \mathrm{H}), 7.40(\mathrm{~d}, J=7.1 \mathrm{~Hz}, 2 \mathrm{H}), 7.34 \sim 7.26(\mathrm{~m}, 3 \mathrm{H}), 7.13 \sim$ $7.09(\mathrm{~m}, 1 \mathrm{H}), 6.87 \sim 6.84(\mathrm{~m}, 2 \mathrm{H}), 6.70 \sim 6.66(\mathrm{~m}, 1 \mathrm{H})$, $4.60(\mathrm{~s}, 1 \mathrm{H}), 2.44 \sim 2.38(\mathrm{~m}, 2 \mathrm{H}), 2.26(\mathrm{~s}, 3 \mathrm{H}), 1.53 \sim 1.52$ (m, 2H), 1.29 (dd, $J=14.4,7.3 \mathrm{~Hz}, 2 \mathrm{H}), 0.87$ (t, $J=7.3$ $\mathrm{Hz}, 3 \mathrm{H}) ;{ }^{13} \mathrm{C} \mathrm{NMR}\left(\mathrm{CDCl}_{3}, 100 \mathrm{MHz}\right) \delta: 157.33,139.00$, $129.03,128.96,128.65,128.45,127.89,125.57,118.90$, 116.84, 75.63, 54.49, 38.94, 29.10, 20.38, 13.92; HRMS (ESI) calcd for $\mathrm{C}_{18} \mathrm{H}_{24} \mathrm{NO}[\mathrm{M}+\mathrm{H}]^{+}$270.1852, found 270.1855 .

2-氟-6-[吗啉(苯基)甲基]苯酚(3w)：白色固体，产率 82\%. m.p. $135.3 \sim 137.2{ }^{\circ} \mathrm{C} ;{ }^{1} \mathrm{H}$ NMR (400 MHz, $\mathrm{CDCl}_{3}$ ) $\delta: 12.19$ (s, 1H), 7.31 (ddd, $J=27.4,18.3,7.0 \mathrm{~Hz}, 5 \mathrm{H}$ ), $7.05 \sim 6.78(\mathrm{~m}, 1 \mathrm{H}), 6.65$ (ddd, $J=17.7,12.6,6.3 \mathrm{~Hz}, 2 \mathrm{H}$ ), $4.47(\mathrm{~s}, 1 \mathrm{H}), 3.76 \sim 3.73(\mathrm{~m}, 4 \mathrm{H}), 2.58 \sim 2.43(\mathrm{~m}, 4 \mathrm{H}) ;{ }^{13} \mathrm{C}$ NMR $\left(\mathrm{CDCl}_{3}, 100 \mathrm{MHz}\right) \delta$ : $151.64(\mathrm{~d}, J=241.9 \mathrm{~Hz})$, 144.66 (d, $J=12.6 \mathrm{~Hz}), 138.63,129.07,128.56,128.41$, 127.11 (d, $J=2.3 \mathrm{~Hz}), 124.34$ (d, $J=3.1 \mathrm{~Hz}), 119.15$ (d, $J=7.1 \mathrm{~Hz}), 115.19$ (d, $J=18 \mathrm{~Hz}), 76.51,66.82,52.19$; HRMS (ESI) calcd for $\mathrm{C}_{17} \mathrm{H}_{19} \mathrm{FNO}_{2}[\mathrm{M}+\mathrm{H}]^{+}$288.1394, found 288.1395 .

2-氟-6-[苯基(硫代吗啉)甲基]苯酚(3x)：白色固体， 产率 84\%. m.p. $121.3 \sim 122.1{ }^{\circ} \mathrm{C}$; ${ }^{1} \mathrm{H}$ NMR $(400 \mathrm{MHz}$, $\left.\mathrm{CDCl}_{3}\right) \delta: 12.26(\mathrm{~s}, 1 \mathrm{H}), 7.59 \sim 7.16(\mathrm{~m}, 5 \mathrm{H}), 6.93(\mathrm{t}, J=$ $9.1 \mathrm{~Hz}, 1 \mathrm{H}), 6.62(\mathrm{dt}, J=7.6,6.0 \mathrm{~Hz}, 2 \mathrm{H}), 4.63(\mathrm{~s}, 1 \mathrm{H})$, $3.26 \sim 2.33(\mathrm{~m}, 8 \mathrm{H}) ;{ }^{13} \mathrm{C}$ NMR $\left(\mathrm{CDCl}_{3}, 100 \mathrm{MHz}\right) \delta$ : 
$151.63(\mathrm{~d}, J=241.9 \mathrm{~Hz}), 145.18$ (d, $J=12.7 \mathrm{~Hz}), 137.60$, 129.03, 128.94, 128.48, 126.91 (d, $J=2.3 \mathrm{~Hz}), 124.40$ (d, $J=2.9 \mathrm{~Hz}), 118.83$ (d, $J=7.1 \mathrm{~Hz}), 115.22$ (d, $J=18 \mathrm{~Hz})$, 76.09, 53.20, 28.06; HRMS (ESI) calcd for $\mathrm{C}_{17} \mathrm{H}_{19}$ FNOS $[\mathrm{M}+\mathrm{H}]^{+}$304.1166, found 304.1168.

4-氟-2-[苯基(硫代吗啉)甲基]苯酚(3y): 白色固体, 产率 80\%. m.p. $140.3 \sim 142.1{ }^{\circ} \mathrm{C}$; ${ }^{1} \mathrm{H}$ NMR $(400 \mathrm{MHz}$, $\left.\mathrm{CDCl}_{3}\right) \delta: 11.57(\mathrm{~s}, 1 \mathrm{H}), 7.42 \sim 7.25(\mathrm{~m}, 5 \mathrm{H}), 6.89 \sim 6.72$ $(\mathrm{m}, 2 \mathrm{H}), 6.60(\mathrm{dd}, J=9.1,2.6 \mathrm{~Hz}, 1 \mathrm{H}), 4.54(\mathrm{~s}, 1 \mathrm{H})$, $3.12 \sim 2.48(\mathrm{~m}, 8 \mathrm{H}) ;{ }^{13} \mathrm{C} \mathrm{NMR}\left(\mathrm{CDCl}_{3}, 100 \mathrm{MHz}\right) \delta$ : 156.05 (d, $J=235.3 \mathrm{~Hz}), 152.66,137.50,129.04,128.91$, 128.48, 125.44 (d, $J=6.7 \mathrm{~Hz}), 117.74$ (d, $J=7.7 \mathrm{~Hz})$, 115.74 (d, $J=23.8 \mathrm{~Hz}), 115.17$ (d, $J=22.5 \mathrm{~Hz}), 75.95$, 53.13, 28.07; HRMS (ESI) calcd for $\mathrm{C}_{17} \mathrm{H}_{19} \mathrm{FNOS}[\mathrm{M}+$ $\mathrm{H}]^{+}$304.1166, found 304.1165.

2-[(苄胺)(苯基)甲基]苯酚(5a): 无色粘稠油状液体, 产率 90\%. ${ }^{1} \mathrm{H}$ NMR (400 MHz, DMSO- $\left.d_{6}\right) \delta: 11.19(\mathrm{~s}$, $1 \mathrm{H}), 7.42(\mathrm{~d}, J=7.4 \mathrm{~Hz}, 2 \mathrm{H}), 7.35 \sim 7.30(\mathrm{~m}, 6 \mathrm{H}), 7.27 \sim$ $7.21(\mathrm{~m}, 2 \mathrm{H}), 7.08 \sim 7.04(\mathrm{~m}, 2 \mathrm{H}), 6.73(\mathrm{dd}, J=13.5,7.4$ $\mathrm{Hz}, 2 \mathrm{H}), 5.01(\mathrm{~s}, 1 \mathrm{H}), 3.66(\mathrm{~s}, 2 \mathrm{H}) ;{ }^{13} \mathrm{C}$ NMR (DMSO- $d_{6}$, $100 \mathrm{MHz}) \delta: 156.85,143.24,140.07,128.78,128.71$, $128.69,128.34,128.23,127.97,127.92,127.41,127.34$, 119.29, 116.28, 62.83, 51.15; HRMS (ESI) calcd for $\mathrm{C}_{20} \mathrm{H}_{20} \mathrm{NO}[\mathrm{M}+\mathrm{H}]^{+}$290.1539, found 290.1540.

2-\{[(2-甲基苄基)胺 $]$ (苯基)甲基 $\}$ 苯酚 $(5 \mathbf{b})$ : 无色粘 稠油状液体, 产率 92\%. ${ }^{1} \mathrm{H}$ NMR (400 MHz, DMSO- $\left.d_{6}\right)$ $\delta: 11.13(\mathrm{~s}, 1 \mathrm{H}), 7.46(\mathrm{~d}, J=7.3 \mathrm{~Hz}, 2 \mathrm{H}), 7.34 \sim 7.31(\mathrm{~m}$, $3 \mathrm{H}), 7.23$ (t, $J=7.3 \mathrm{~Hz}, 1 \mathrm{H}), 7.17 \sim 7.12(\mathrm{~m}, 3 \mathrm{H}), 7.07 \sim$ $7.04(\mathrm{~m}, 2 \mathrm{H}), 6.76(\mathrm{dd}, J=6.3,2.3 \mathrm{~Hz}, 1 \mathrm{H}), 6.71(\mathrm{t}, J=7.4$ $\mathrm{Hz}, 1 \mathrm{H}), 5.09$ (s, 1H), 3.64 (s, 2H), 2.20 (s, 3H); ${ }^{13} \mathrm{C}$ NMR $\left(\mathrm{DMSO}-d_{6}, 100 \mathrm{MHz}\right) \delta: 156.80,143.24,138.11,136.52$, $130.44,129.09,128.78,128.67,128.61,128.34,128.03$, $127.43,127.34,126.23,119.25,116.24,63.15,48.85$, 19.05; HRMS (ESI) calcd for $\mathrm{C}_{21} \mathrm{H}_{22} \mathrm{NO}[\mathrm{M}+\mathrm{H}]^{+}$ 304.1696, found 304.1698.

2-[(芰胺)(间甲基)甲基]苯酚(5c)：无色粘稠油状液 体, 产率 93\%. ${ }^{1} \mathrm{H}$ NMR (400 MHz, DMSO- $d_{6}$ ) $\delta: 11.26$ $(\mathrm{s}, 1 \mathrm{H}), 7.33 \sim 7.30(\mathrm{~m}, 4 \mathrm{H}), 7.27 \sim 7.19(\mathrm{~m}, 4 \mathrm{H}), 7.05(\mathrm{t}$, $J=7.5 \mathrm{~Hz}, 3 \mathrm{H}), 6.72(\mathrm{t}, J=8.3 \mathrm{~Hz}, 2 \mathrm{H}), 4.96$ (s, 1H), 3.65 $(\mathrm{s}, 2 \mathrm{H}), 2.27(\mathrm{~s}, 3 \mathrm{H}) ;{ }^{13} \mathrm{C}$ NMR (DMSO- $\left.d_{6}, 100 \mathrm{MHz}\right) \delta$ : 156.84, 143.16, 140.02, 137.78, 129.14, 128.74, 128.69, 128.44,. 128.29, 128.18, 128.07, 127.33, 124.97, 119.26, $116.25,62.86,51.12,21.62$; HRMS (ESI) calcd for $\mathrm{C}_{21} \mathrm{H}_{22} \mathrm{NO}[\mathrm{M}+\mathrm{H}]^{+}$304.1696, found 304.1696.

2-[(芐胺)(邻甲基)甲基]苯酚(5d): 无色粘稠油状液
体, 产率 92\%. ${ }^{1} \mathrm{H}$ NMR (400 MHz, DMSO- $\left.d_{6}\right) \delta: 11.28$ (s, $1 \mathrm{H}), 7.42$ (d, $J=7.2 \mathrm{~Hz}, 1 \mathrm{H}), 7.35 \sim 7.24(\mathrm{~m}, 5 \mathrm{H}), 7.21 \sim$ $7.11(\mathrm{~m}, 3 \mathrm{H}), 7.11 \sim 7.02(\mathrm{~m}, 1 \mathrm{H}), 6.91 \sim 6.82(\mathrm{~m}, 1 \mathrm{H})$, $6.77(\mathrm{~d}, J=7.5 \mathrm{~Hz}, 1 \mathrm{H}), 6.70(\mathrm{t}, J=7.4 \mathrm{~Hz}, 1 \mathrm{H}), 5.20$ (s, $1 \mathrm{H}), 3.66$ (s, 2H), $2.16(\mathrm{~s}, 3 \mathrm{H}) ;{ }^{13} \mathrm{C}$ NMR (DMSO- $d_{6}, 100$ $\mathrm{MHz}) \delta: 157.29,140.63,140.01,136.45,130.67,128.82$, $128.77,128.67,128.36,127.68,127.61,127.38,127.24$, 126.51, 119.19, 116.19, 58.07, 51.20, 19.26; HRMS (ESI) calcd for $\mathrm{C}_{21} \mathrm{H}_{22} \mathrm{NO}[\mathrm{M}+\mathrm{H}]^{+}$304.1696, found 304.1698.

2-\{[(4-甲基苄基)胺](苯基)甲基 $\}$ 苯酚(5e)：无色粘 稠油状液体，产率 94\%. ${ }^{1} \mathrm{H}$ NMR (400 MHz, DMSO- $d_{6}$ ) $\delta: 11.29$ (s, 1H), 7.40 (d, $J=7.3 \mathrm{~Hz}, 2 \mathrm{H}), 7.31(\mathrm{t}, J=7.5$ $\mathrm{Hz}, 2 \mathrm{H}), 7.21$ (dd, $J=16.2,7.6 \mathrm{~Hz}, 3 \mathrm{H}), 7.13$ (d, $J=7.9$ $\mathrm{Hz}, 2 \mathrm{H}), 7.05$ (dd, $J=7.0,5.5 \mathrm{~Hz}, 2 \mathrm{H}), 6.72$ (dd, $J=13.6$, $7.4 \mathrm{~Hz}, 2 \mathrm{H}), 4.99$ (s, 1H), 3.61 (s, 2H), 2.28 (s, 3H); ${ }^{13} \mathrm{C}$ NMR (DMSO- $\left.d_{6}, 100 \mathrm{MHz}\right) \delta: 156.90,143.16,136.87$, $136.38,129.29,128.78,128.73,128.67,128.33,128.10$, 127.91, 127.42, 119.25, 116.28, 62.78, 50.77, 21.18; HRMS (ESI) calcd for $\mathrm{C}_{21} \mathrm{H}_{22} \mathrm{NO}[\mathrm{M}+\mathrm{H}]^{+}$304.1696, found 304.1696 .

2-\{[(4-甲基苄基)胺](苯基)甲基 $\}$ 苯酚(5f)：无色粘 稠油状液体, 产率 90\%. ${ }^{1} \mathrm{H}$ NMR (400 MHz, DMSO- $d_{6}$ ) $\delta: 11.27(\mathrm{~s}, 1 \mathrm{H}), 7.43$ (d, $J=7.4 \mathrm{~Hz}, 2 \mathrm{H}), 7.31(\mathrm{t}, J=7.5$ $\mathrm{Hz}, 2 \mathrm{H}), 7.21(\mathrm{dd}, J=14.3,7.2 \mathrm{~Hz}, 2 \mathrm{H}), 7.13 \sim 7.04(\mathrm{~m}$, $5 \mathrm{H}), 6.77 \sim 6.70(\mathrm{~m}, 2 \mathrm{H}), 5.02(\mathrm{~s}, 1 \mathrm{H}), 3.63(\mathrm{~s}, 2 \mathrm{H}), 2.28$ $(\mathrm{s}, 3 \mathrm{H}) ;{ }^{13} \mathrm{C}$ NMR (DMSO- $\left.d_{6}, 100 \mathrm{MHz}\right) \delta: 156.9,143.22$, $139.93,137.75,129.35,128.79,128.74,128.62,128.35$, $128.20,127.98,127.94,127.42,125.77,119.28,116.30$, 63.01, 51.19, 21.53; HRMS (ESI) calcd for $\mathrm{C}_{21} \mathrm{H}_{22} \mathrm{NO}[\mathrm{M}$ $+\mathrm{H}]^{+}$304.1696, found 304.1695.

2-[(芐胺)(苯基)甲基]-4-甲基苯酚(5g)：无色粘稠油 状液体, 产率 90\%. ${ }^{1} \mathrm{H}$ NMR (400 MHz, DMSO- $\left.d_{6}\right) \delta$ : 10.97 (s, 1H), 7.42 (d, $J=7.4 \mathrm{~Hz}, 2 \mathrm{H}), 7.31$ (dd, $J=8.8$, $6.1 \mathrm{~Hz}, 6 \mathrm{H}), 7.26 \sim 7.21(\mathrm{~m}, 2 \mathrm{H}), 6.90(\mathrm{~s}, 1 \mathrm{H}), 6.90 \sim 6.84$ $(\mathrm{m}, 1 \mathrm{H}), 6.65(\mathrm{~d}, J=8.1 \mathrm{~Hz}, 1 \mathrm{H}), 4.98(\mathrm{~s}, 1 \mathrm{H}), 3.66(\mathrm{~s}$, 2H), $2.13(\mathrm{~s}, 3 \mathrm{H}) ;{ }^{13} \mathrm{C}$ NMR (DMSO- $\left.d_{6}, 100 \mathrm{MHz}\right) \delta$ : $154.47,143.42,140.10,129.13,128.77,128.71,128.68$, $128.61,127.88,127.81,127.64,127.37,127.32,116.16$, 63.03, 51.22, 20.76; HRMS (ESI) calcd for $\mathrm{C}_{21} \mathrm{H}_{22} \mathrm{NO}$ $[\mathrm{M}+\mathrm{H}]^{+}$304.1696, found 304.1698.

2-[(芐胺)(对甲苯基)甲基]苯酚(5h): 无色粘稠油状 液体, 产率 95\%. ${ }^{1} \mathrm{H}$ NMR (400 MHz, DMSO- $d_{6}$ ) $\delta: 11.30$ $(\mathrm{s}, 1 \mathrm{H}), 7.35 \sim 7.25(\mathrm{~m}, 7 \mathrm{H}), 7.12(\mathrm{~d}, J=7.9 \mathrm{~Hz}, 2 \mathrm{H})$, $7.14 \sim 7.12(\mathrm{~m}, 2 \mathrm{H}), 6.73 \sim 6.68(\mathrm{~m}, 2 \mathrm{H}), 4.96(\mathrm{~s}, 1 \mathrm{H})$, $3.64(\mathrm{~s}, 2 \mathrm{H}), 2.26(\mathrm{~s}, 3 \mathrm{H}) ;{ }^{13} \mathrm{C}$ NMR (DMSO- $\left.d_{6}, 100 \mathrm{MHz}\right)$ 
$\delta: 156.90,140.07,140.01,136.51,129.35,128.81,128.71$, $128.64,128.62,128.27,127.84,127.34,119.20,116.24$, 62.67, 51.05, 21.12; HRMS (ESI) calcd for $\mathrm{C}_{21} \mathrm{H}_{22} \mathrm{NO}$ $[\mathrm{M}+\mathrm{H}]^{+}$304.1696, found 304.1693.

2-[(芐胺)(苯基)甲基]-6-(叔丁基)苯酚(5i)：无色粘 稠油状液体, 产率 $92 \% .{ }^{1} \mathrm{H}$ NMR (400 MHz, DMSO- $d_{6}$ ) $\delta: 13.05$ (s, $1 \mathrm{H}), 7.45$ (d, $J=7.3 \mathrm{~Hz}, 2 \mathrm{H}), 7.37 \sim 7.31(\mathrm{~m}$, $6 \mathrm{H}), 7.29 \sim 7.26(\mathrm{~m}, 2 \mathrm{H}), 7.07(\mathrm{dd}, J=6.0,3.4 \mathrm{~Hz}, 1 \mathrm{H})$, $6.60 \sim 6.58(\mathrm{~m}, 2 \mathrm{H}), 4.95(\mathrm{~s}, 1 \mathrm{H}), 4.23(\mathrm{~s}, 1 \mathrm{H}), 3.68(\mathrm{~d}, J=$ $4.3 \mathrm{~Hz}, 2 \mathrm{H}$ ), 1.39 (s, 9H); ${ }^{13} \mathrm{C}$ NMR (DMSO- $d_{6}, 100 \mathrm{MHz}$ ) $\delta: 157.19,141.86,139.13,136.37,129.00,128.88,128.81$, $128.18,127.85,127.61,127.35,126.26,125.65,118.30$, 66.04, 50.98, 34.88, 29.93; HRMS (ESI) calcd for $\mathrm{C}_{24} \mathrm{H}_{28} \mathrm{NO}[\mathrm{M}+\mathrm{H}]^{+}$346.2165, found 346.2165.

2-[(芐胺)(苯基)甲基]-6-乙氧基苯酚 $(\mathbf{5} \mathbf{j}$ ): 无色粘稠 油状液体, 产率 $89 \% .{ }^{1} \mathrm{H}$ NMR (400 MHz, DMSO- $\left.d_{6}\right) \delta$ : 10.86 (s, 1H), 7.41 (d, $J=7.3 \mathrm{~Hz}, 2 \mathrm{H}), 7.31$ (dd, $J=10.4$, $5.7 \mathrm{~Hz}, 6 \mathrm{H}), 7.24$ (dd, $J=11.1,5.8 \mathrm{~Hz}, 2 \mathrm{H}), 6.78$ (dd, $J=$ $7.4,1.9 \mathrm{~Hz}, 1 \mathrm{H}), 6.70 \sim 6.63(\mathrm{~m}, 2 \mathrm{H}), 5.02(\mathrm{~s}, 1 \mathrm{H}), 3.98(\mathrm{q}$, $J=6.9 \mathrm{~Hz}, 2 \mathrm{H}), 3.66$ (d, $J=5.3 \mathrm{~Hz}, 2 \mathrm{H}), 3.37$ (s, 1H), 1.32 (t, $J=6.9 \mathrm{~Hz}, 3 \mathrm{H}$ ); ${ }^{13} \mathrm{C}$ NMR (DMSO- $\left.d_{6}, 100 \mathrm{MHz}\right) \delta$ : $147.28,146.44,143.21,139.99,128.75,128.71,128.67$, $128.36,127.86,127.41,127.34,120.65,118.88,112.48$, 64.31, 62.94, 51.11, 15.30; HRMS (ESI) calcd for $\mathrm{C}_{22} \mathrm{H}_{24} \mathrm{NO}_{2}[\mathrm{M}+\mathrm{H}]^{+}$334.1802, found 334.1805.

2-\{[1,1'-二苯基]-4-基(芐胺)甲基\}苯酚(5k): 无色粘 稠油状液体, 产率 88\%. ${ }^{1} \mathrm{H}$ NMR (400 MHz, DMSO- $d_{6}$ ) $\delta: 11.15$ (s, 1H), 7.62 (t, $J=8.2 \mathrm{~Hz}, 4 \mathrm{H}), 7.50$ (d, $J=8.2$ $\mathrm{Hz}, 2 \mathrm{H}), 7.44(\mathrm{t}, J=7.6 \mathrm{~Hz}, 2 \mathrm{H}), 7.36 \sim 7.32(\mathrm{~m}, 5 \mathrm{H}), 7.26$ (dd, $J=8.6,4.2 \mathrm{~Hz}, 1 \mathrm{H}), 7.16(\mathrm{~d}, J=6.9 \mathrm{~Hz}, 1 \mathrm{H}), 7.19 \sim$ 7.05 (m, 1H), 6.75 (t, $J=8.0 \mathrm{~Hz}, 2 \mathrm{H}), 5.07$ (s, 1H), 3.69 (s, $2 \mathrm{H}$ ); ${ }^{13} \mathrm{C}$ NMR (DMSO- $\left.d_{6}, 100 \mathrm{MHz}\right) \delta: 156.72,142.59$, $140.46,140.13,139.33,129.37,128.72,128.67,128.46$, $128.35,128.27,127.78,127.33,127.12,127.07,119.36$, 116.27, 62.30, 51.15; HRMS (ESI) calcd for $\mathrm{C}_{26} \mathrm{H}_{24} \mathrm{NO}$ $[\mathrm{M}+\mathrm{H}]^{+} 366.1852$, found 366.1855 .

2-[(苄胺)(4-氯苯基)甲基]苯酚(51): 无色粘稠油状 液体, 产率 84\%. ${ }^{1} \mathrm{H}$ NMR (400 MHz, DMSO- $\left.d_{6}\right) \delta: 10.84$ (s, 1H), 7.44 (d, $J=8.5 \mathrm{~Hz}, 2 \mathrm{H}), 7.37$ (d, $J=8.5 \mathrm{~Hz}, 2 \mathrm{H})$, $7.32(\mathrm{~d}, J=4.3 \mathrm{~Hz}, 4 \mathrm{H}), 7.25(\mathrm{dd}, J=8.8,4.5 \mathrm{~Hz}, 1 \mathrm{H})$, $7.14(\mathrm{~d}, J=7.6 \mathrm{~Hz}, 1 \mathrm{H}), 7.06$ (dd, $J=11.2,4.1 \mathrm{~Hz}, 1 \mathrm{H})$, $6.77 \sim 6.73(\mathrm{~m}, 2 \mathrm{H}), 5.04(\mathrm{~s}, 1 \mathrm{H}), 3.64(\mathrm{~s}, 2 \mathrm{H}) ;{ }^{13} \mathrm{C} \mathrm{NMR}$ (DMSO- $\left.d_{6}, 100 \mathrm{MHz}\right) \delta: 156.41,142.52,140.19,131.83$, $129.77,128.69,128.67,128.63,128.44,128.42,128.30$, 127.30, 119.43, 116.21, 61.42, 51.14; HRMS (ESI) calcd for $\mathrm{C}_{20} \mathrm{H}_{19} \mathrm{ClNO}[\mathrm{M}+\mathrm{H}]^{+}$324.1150, found 324.1152.

2-[(芐胺)(3-氟苯基)甲基]苯酚(5m): 无色粘稠油状 液体, 产率 $82 \% .{ }^{1} \mathrm{H}$ NMR $\left(400 \mathrm{MHz}\right.$, DMSO- $\left.d_{6}\right) \delta: 10.83$ (s, $1 \mathrm{H}), 7.38 \sim 7.30(\mathrm{~m}, 5 \mathrm{H}), 7.28 \sim 7.22(\mathrm{~m}, 3 \mathrm{H}), 7.16(\mathrm{~d}$, $J=7.5 \mathrm{~Hz}, 1 \mathrm{H}), 7.06(\mathrm{dt}, J=12.0,5.5 \mathrm{~Hz}, 2 \mathrm{H}), 6.77(\mathrm{dd}$, $J=14.6,7.6 \mathrm{~Hz}, 2 \mathrm{H}), 5.08(\mathrm{~s}, 1 \mathrm{H}), 3.66(\mathrm{~s}, 2 \mathrm{H}) ;{ }^{13} \mathrm{C} \mathrm{NMR}$ (DMSO- $\left.d_{6}, 100 \mathrm{MHz}\right) \delta: 162.73$ (d, $\left.J=241.6 \mathrm{~Hz}\right), 156.41$, 146.59 (d, $J=6.6 \mathrm{~Hz}), 140.19,130.59$ (d, $J=8.1 \mathrm{~Hz})$, $128.71(\mathrm{~d}, J=2.4 \mathrm{~Hz}), 128.66,128.63,128.47,128.29$, 127.30, $124.02(\mathrm{~d}, J=2.5 \mathrm{~Hz}), 119.46,116.24,114.42(\mathrm{~d}$, $J=21.5 \mathrm{~Hz}), 114.04$ (d, $J=20.9 \mathrm{~Hz}), 61.57,51.16$; HRMS (ESI) calcd for $\mathrm{C}_{20} \mathrm{H}_{19} \mathrm{FNO}[\mathrm{M}+\mathrm{H}]^{+}$308.1445, found 308.1446 .

2-\{[(2,6-二氯苄基)胺](苯基)甲基 $\}$ 苯酚 $(\mathbf{5 n})$ : 无色 粘稠油状液体, 产率 $82 \% .{ }^{1} \mathrm{H}$ NMR $(400 \mathrm{MHz}$, DMSO$\left.d_{6}\right) \delta: 10.50(\mathrm{~s}, 1 \mathrm{H}), 7.47(\mathrm{~d}, J=7.3 \mathrm{~Hz}, 2 \mathrm{H}), 7.43(\mathrm{~d}, J=$ $8.1 \mathrm{~Hz}, 2 \mathrm{H}), 7.35 \sim 7.22(\mathrm{~m}, 4 \mathrm{H}), 7.07 \sim 7.01(\mathrm{~m}, 2 \mathrm{H}), 6.78$ (d, $J=7.9 \mathrm{~Hz}, 1 \mathrm{H}), 6.71$ (t, $J=7.4 \mathrm{~Hz}, 1 \mathrm{H}), 5.15(\mathrm{~s}, 1 \mathrm{H})$, 3.90 (s, 2H), 3.06 (s, 1H); ${ }^{13} \mathrm{C}$ NMR (DMSO- $d_{6}, 100 \mathrm{MHz}$ ) $\delta: 156.41,142.85,135.93,135.44,130.33,128.96,128.84$, $128.68,128.47,128.36,128.15,127.45,119.17,116.05$, 62.72, 47.10; HRMS (ESI) calcd for $\mathrm{C}_{20} \mathrm{H}_{18} \mathrm{Cl}_{2} \mathrm{NO}[\mathrm{M}+\mathrm{H}]$ ${ }^{+} 358.0760$, found 358.0762 .

2-\{[(4-碘苄基)胺](苯基)甲基 $\}$ 苯酚(50): 无色粘稠 油状液体, 产率 $82 \% .{ }^{1} \mathrm{H}$ NMR $\left(400 \mathrm{MHz}, \mathrm{DMSO}-d_{6}\right) \delta$ : 10.92 (s, 1H), 7.68 (d, $J=8.2 \mathrm{~Hz}, 2 \mathrm{H}), 7.41$ (d, $J=7.3 \mathrm{~Hz}$, $2 \mathrm{H}), 7.30(\mathrm{t}, J=7.5 \mathrm{~Hz}, 2 \mathrm{H}), 7.21(\mathrm{t}, J=7.3 \mathrm{~Hz}, 1 \mathrm{H})$, $7.14 \sim 7.11(\mathrm{~m}, 3 \mathrm{H}), 7.07 \sim 7.03(\mathrm{~m}, 1 \mathrm{H}), 6.76 \sim 6.71(\mathrm{~m}$, $2 \mathrm{H}), 5.00(\mathrm{~s}, 1 \mathrm{H}), 3.60(\mathrm{~s}, 2 \mathrm{H}) ;{ }^{13} \mathrm{C}$ NMR (DMSO- $d_{6}, 100$ MHz) $\delta: 156.56,143.33,140.07,137.43,131.06,128.75$, $128.61,128.39,128.30,127.88,127.36,119.36,116.21$, 93.06, 62.23, 50.52; HRMS (ESI) calcd for $\mathrm{C}_{20} \mathrm{H}_{19} \mathrm{INO}$ $[\mathrm{M}+\mathrm{H}]^{+}$416.0506, found 416.0505.

2-\{[(4-溴芐基)胺](苯基)甲基 $\}$ 苯酚 (5p): 无色粘稠 油状液体, 产率 $80 \% .{ }^{1} \mathrm{H}$ NMR $\left(400 \mathrm{MHz}, \mathrm{DMSO}-d_{6}\right) \delta$ : 10.94 (s, 1H), 7.50 (d, $J=8.3 \mathrm{~Hz}, 2 \mathrm{H}), 7.42$ (d, $J=7.3 \mathrm{~Hz}$, 2H), 7.29 (dd, $J=15.3,7.8 \mathrm{~Hz}, 4 \mathrm{H}), 7.21(\mathrm{t}, J=7.3 \mathrm{~Hz}$, $1 \mathrm{H}), 7.14 \sim 7.12(\mathrm{~m}, 1 \mathrm{H}), 7.18 \sim 7.03(\mathrm{~m}, 1 \mathrm{H}), 6.78 \sim 6.71$ $(\mathrm{m}, 2 \mathrm{H}), 5.02(\mathrm{~s}, 1 \mathrm{H}), 3.63(\mathrm{~s}, 2 \mathrm{H}) ;{ }^{13} \mathrm{C}$ NMR (DMSO- $d_{6}$, $100 \mathrm{MHz}) \delta$ : 156.61, 143.34, 139.68, 131.56, 130.87, $128.76,128.64,128.38,128.33,127.89,127.37,120.38$, 119.37, 116.25, 62.33, 50.45; HRMS (ESI) calcd for $\mathrm{C}_{20} \mathrm{H}_{19} \mathrm{INO}[\mathrm{M}+\mathrm{H}]^{+}$416.0506, found 416.0505.

2-\{[(4-氟芐基)胺](苯基)甲基 $\}$ 苯酚 (5q)：无色粘稠 油状液体, 产率 $76 \%$. ${ }^{1} \mathrm{H}$ NMR $\left(400 \mathrm{MHz}, \mathrm{DMSO}-d_{6}\right) \delta$ : 
$11.07(\mathrm{~s}, 1 \mathrm{H}), 7.42(\mathrm{~d}, J=7.3 \mathrm{~Hz}, 2 \mathrm{H}), 7.37 \sim 7.20(\mathrm{~m}$, $6 \mathrm{H}), 7.16 \sim 7.04(\mathrm{~m}, 5 \mathrm{H}), 6.74(\mathrm{dd}, J=15.4,7.8 \mathrm{~Hz}, 2 \mathrm{H})$, $5.02(\mathrm{~s}, 1 \mathrm{H}), 3.65(\mathrm{~s}, 2 \mathrm{H}) ;{ }^{13} \mathrm{C}$ NMR (DMSO- $\left.d_{6}, 100 \mathrm{MHz}\right)$ $\delta: 161.73$ (d, $J=240.8 \mathrm{~Hz}), 156.70,143.31,136.30$ (d, $J=$ $2.9 \mathrm{~Hz}), 130.57$ (d, $J=8.0 \mathrm{~Hz}), 128.763,128.714,128.67$, $128.31,127.89$, 127.38, 119.33, 116.25, 115.49, 115.28, 62.51, 50.36; HRMS (ESI) calcd for $\mathrm{C}_{20} \mathrm{H}_{19} \mathrm{FNO}[\mathrm{M}+\mathrm{H}]^{+}$ 308.1445 , found 308.1445 .

2-[(苄胺)(环己基)甲基]苯酚(5r)：无色粘稠油状液 体, 产率 82\%. ${ }^{1} \mathrm{H}$ NMR (400 MHz, DMSO- $\left.d_{6}\right) \delta: 11.73$ (s, $1 \mathrm{H}), 7.34 \sim 7.23(\mathrm{~m}, 5 \mathrm{H}), 7.07(\mathrm{td}, J=8.0,1.5 \mathrm{~Hz}, 1 \mathrm{H})$, $6.91 \sim 6.89(\mathrm{~m}, 1 \mathrm{H}), 6.74 \sim 6.69(\mathrm{~m}, 2 \mathrm{H}), 3.68(\mathrm{~d}, J=13.5$ $\mathrm{Hz}, 1 \mathrm{H}), 3.50 \sim 3.37(\mathrm{~m}, 4 \mathrm{H}), 1.86(\mathrm{~d}, J=12.5 \mathrm{~Hz}, 1 \mathrm{H})$, $1.6 \sim 1.56(\mathrm{~m}, 4 \mathrm{H}), 1.43(\mathrm{~d}, J=12.5 \mathrm{~Hz}, 1 \mathrm{H}), 1.11 \sim 0.88$ $(\mathrm{m}, 5 \mathrm{H}) ;{ }^{13} \mathrm{C}$ NMR (DMSO- $\left.d_{6}, 100 \mathrm{MHz}\right) \delta$ : 158.03, $139.74,130.23,128.70,128.66,128.14,127.35,125.01$, 118.59 , 116.16, 66.42, 51.08, 43.17, 29.87, 29.50, 26.42, 26.33, 26.25; HRMS (ESI) calcd for $\mathrm{C}_{20} \mathrm{H}_{26} \mathrm{FNO}[\mathrm{M}+\mathrm{H}]^{+}$ 296.2009, found 296.2010.

2-\{苯基[(噻吩-2-基甲基)胺]甲基 $\}$ 苯酚(5s)：无色粘 稠油状液体, 产率 93\%. ${ }^{1} \mathrm{H}$ NMR (400 MHz, DMSO- $\left.d_{6}\right)$ $\delta$ : $10.65(\mathrm{~s}, 1 \mathrm{H}), 7.40(\mathrm{t}, J=5.5 \mathrm{~Hz}, 3 \mathrm{H}), 7.31$ (t, $J=7.5$ $\mathrm{Hz}, 2 \mathrm{H}), 7.21(\mathrm{t}, J=7.3 \mathrm{~Hz}, 1 \mathrm{H}), 7.14 \sim 7.12(\mathrm{~m}, 1 \mathrm{H})$, $7.07 \sim 7.03(\mathrm{~m}, 1 \mathrm{H}), 6.96(\mathrm{dd}, J=4.9,3.5 \mathrm{~Hz}, 1 \mathrm{H}), 6.92(\mathrm{~d}$, $J=3.0 \mathrm{~Hz}, 1 \mathrm{H}), 6.75(\mathrm{dd}, J=14.2,7.4 \mathrm{~Hz}, 2 \mathrm{H}), 5.09$ (s, $1 \mathrm{H}), 3.83(\mathrm{~s}, 2 \mathrm{H}) ;{ }^{13} \mathrm{C}$ NMR (DMSO-d, $\left.100 \mathrm{MHz}\right) \delta$ : $156.45,143.90,143.26,128.72,128.57,128.50,128.31$, 127.95, 127.33, 127.23, 125.70, 125.26, 119.37, 116.17, 61.66, 45.75; HRMS (ESI) calcd for $\mathrm{C}_{18} \mathrm{H}_{18} \mathrm{NOS}[\mathrm{M}+\mathrm{H}]^{+}$ 296.1104, found 296.1105.

2 - $\{[$ (3-氯茮基)胺 $]$ (苯基)甲基 $\}$ 苯酚(5t)：无色粘稠 油状液体，产率 $88 \% .{ }^{1} \mathrm{H}$ NMR $\left(400 \mathrm{MHz}, \mathrm{DMSO}-d_{6}\right) \delta$ : 10.85 (s, 1H), 7.43 (d, $J=7.4 \mathrm{~Hz}, 3 \mathrm{H}), 7.32$ (dt, $J=10.8$, $7.6 \mathrm{~Hz}, 4 \mathrm{H}), 7.26 \sim 7.20(\mathrm{~m}, 2 \mathrm{H}), 7.16 \sim 7.14(\mathrm{~m}, 1 \mathrm{H})$, $7.08 \sim 7.04(\mathrm{~m}, 1 \mathrm{H}), 6.75(\mathrm{dd}, J=15.5,7.8 \mathrm{~Hz}, 2 \mathrm{H}), 5.04$ $(\mathrm{s}, 1 \mathrm{H}), 3.66(\mathrm{~s}, 2 \mathrm{H}) ;{ }^{13} \mathrm{C}$ NMR (DMSO- $\left.d_{6}, 100 \mathrm{MHz}\right) \delta$ : $156.50,143.39,142.95,133.47,130.48,128.75,128.58$, $128.39,128.30,127.87,127.87,127.35,127.28,127.21$, 119.39, 116.21, 62.20, 50.58; HRMS (ESI) calcd for $\mathrm{C}_{20} \mathrm{H}_{19} \mathrm{CINO}[\mathrm{M}+\mathrm{H}]^{+}$324.1150, found 324.1150.

2-[(苄胺)(苯基)甲基]-3-氟苯酚 $(5 \mathbf{u})$ ：无色粘稠油状 液体, 产率 $81 \% .{ }^{1} \mathrm{H}$ NMR (400 MHz, DMSO- $\left.d_{6}\right) \delta: 10.73$ (s, 1H), 7.44 (d, $J=7.4 \mathrm{~Hz}, 2 \mathrm{H}), 7.33$ (t, $J=6.0 \mathrm{~Hz}, 6 \mathrm{H})$, $7.29 \sim 7.18(\mathrm{~m}, 2 \mathrm{H}), 7.06(\mathrm{dd}, J=9.8,3.0 \mathrm{~Hz}, 1 \mathrm{H}), 6.98 \sim$ $6.83(\mathrm{~m}, 1 \mathrm{H}), 6.75(\mathrm{dd}, J=8.8,4.9 \mathrm{~Hz}, 1 \mathrm{H}), 5.05(\mathrm{~s}, 1 \mathrm{H})$, $3.66(\mathrm{~s}, 2 \mathrm{H}) ;{ }^{13} \mathrm{C}$ NMR (DMSO- $\left.d_{6}, 100 \mathrm{MHz}\right) \delta$ : 156.00 (d, $J=232.1 \mathrm{~Hz}), 152.57,143.07,140.26,130.53$ (d, $J=6.3$ $\mathrm{Hz}), 128.79,128.65$ (d, $J=5.0 \mathrm{~Hz}), 127.87,127.37$ (d, $J=$ $17.7 \mathrm{~Hz}), 116.85,114.71,114.45$ (d, $J=4.4 \mathrm{~Hz}$ ), 114.20; HRMS (ESI) calcd for $\mathrm{C}_{20} \mathrm{H}_{19} \mathrm{FNO}[\mathrm{M}+\mathrm{H}]^{+}$308.1445, found 308.1447 .

2-[(芰胺)(苯基)甲基]-6-氟苯酚(5v)：无色粘稠油状 液体，产率 $82 \% .{ }^{1} \mathrm{H}$ NMR $\left(400 \mathrm{MHz}, \mathrm{DMSO}-d_{6}\right) \delta: 7.44$ $(\mathrm{d}, J=7.4 \mathrm{~Hz}, 2 \mathrm{H}), 7.41 \sim 7.29(\mathrm{~m}, 6 \mathrm{H}), 7.30 \sim 7.16(\mathrm{~m}$, 2H), $7.09 \sim 6.93(\mathrm{~m}, 1 \mathrm{H}), 6.84(\mathrm{~d}, J=7.7 \mathrm{~Hz}, 1 \mathrm{H}), 6.69$ (td, $J=7.9,5.1 \mathrm{~Hz}, 1 \mathrm{H}), 5.09$ (s, 1H), 3.69 (d, $J=0.8 \mathrm{~Hz}$, $2 \mathrm{H}) ;{ }^{13} \mathrm{C}$ NMR (DMSO- $\left.d_{6}, 100 \mathrm{MHz}\right) \delta: 151.58$ (d, $J=$ $238.5 \mathrm{~Hz}), 145.19$ (d, $J=12.9 \mathrm{~Hz}), 142.36,139.52,130.62$ $(\mathrm{d}, J=2.3 \mathrm{~Hz}), 128.97,128.79,128.74,127.96,127.79$, 127.50, 124.07 (d, $J=2.5 \mathrm{~Hz}), 118.84$ (d, $J=7.0 \mathrm{~Hz})$, 114.89 (d, $J=18.0 \mathrm{~Hz}), 63.42$ (d, $J=1.8 \mathrm{~Hz}), 51.05$; HRMS (ESI) calcd for $\mathrm{C}_{20} \mathrm{H}_{19} \mathrm{FNO}[\mathrm{M}+\mathrm{H}]^{+}$308.1445, found 308.1447 .

辅助材料(Supporting Information) 化合物 $\mathbf{3 a} \sim \mathbf{3 y}$ 和 $\mathbf{5 a} \sim \mathbf{5 v}$ 的 ${ }^{1} \mathrm{H}$ NMR 和 ${ }^{13} \mathrm{C}$ NMR 谱图. 这些材料可以免 费从本刊网站(http://sioc-journal.cn/)上下载.

\section{References}

[1] Betti, M. Org. Synth. 1929, 9, 60

[2] Waisser, K.; Gregor, K.; Kubicova, L.; Klimesova, V.; Kunes, J.; Machacek, M. Eur. J. Med. Chem. 2000, 35, 733.

[3] Bouaziz, Z.; Riondel, J.; Mey, A.; Berlion, M.; Villard, J.; Filliond, H. Eur. J. Med. Chem. 1991, 26, 469.

[4] Arthington, S. B.; Motley, A. M.; Warnock, D. W.; Morrison, C. J. J. Clin. Microbiol. 2000, 38, 2254.

[5] Chylinska, J. B.; Urbanski, T.; Mordarski, M. J. Med. Chem. 1963, 6,484

[6] Benameur, L.; Bouaziz, Z.; Nebois, P.; Bartoli, M. H.; Boitard, M.; Chem. Pharm. Bull. 1996, 44, 605 .

[7] Mathew, B. P.; Kumar, A.; Sharma, S.; Shukla, P. K.; Nath, M. Eur. J. Med. Chem. 2010, 45,1502 .

[8] Lu, J.; Xu, X.; Wang, C.; He, J.; Hu, Y.; Hu, H. Tetrahedron. Lett. 2002, 43, 8367.

[9] Feng, J.; Bohle, D. S.; Li, C. J. Tetrahedron: Asymmetry 2007, 18, 1043.

[10] Kidwai, M.; Chauhan, R. Asian J. Org. Chem. 2013, 2, 395.

[11] Safari, J.; Zarnegar, Z. J. Mol. Catal. A: Chem. 2013, 379, 269.

[12] Hajjami, M.; Ghorbani, F.; Bakhti, F. Appl. Catal. A 2014, 470, 303.

[13] Hajipour, A. R.; Ghayeb, Y.; Sheikhan, N.; Ruoho, A. E. Tetrahedron Lett. 2009, 50, 5649.

[14] Pathak, T. P.; Sigman, M. S. J. Org. Chem. 2011, 76, 9210.

[15] Willis, N. J.; Bray, C. D. Chem.-Eur. J. 2012, 18, 9160.

[16] Caruana, L.; Fochi, M.; Bernardi, L. Molecules 2015, 20, 11733.

[17] Wang, Z.; Sun, J. Synthesis 2015, 47, 3629.

[18] Van De Water, R. W.; Pettus, T. R. R. Tetrahedron 2002, 58, 5367.

[19] Kulikov, A.; Arumugam, S.; Popik, V. V. J. Org. Chem. 2008, 73, 7611.

[20] Mattson, A. E.; Scheidt, K. A. J. Am. Chem. Soc. 2007, 129, 4508

[21] Luan, Y.; Schaus, S. E. J. Am. Chem. Soc. 2012, 134, 19965.

[22] Shaikh, A. K.; Cobb, A. J. A.; Varounis, G. Org. Lett. 2012, 14, 584. 
[23] Chen, M. W.; Gao, L. L.; Ye, Z. S.; Jiang, G. F.; Zhou, Y. G. Chem. Commun. 2013, 49, 1660.

[24] Yoshida, H.; Watanabe, M.; Fukushima, H.; Ohshita, J.; Kunai, A. A. Org. Lett. 2004, 6, 4049.

[25] Bai, W. J.; David, J. G.; Feng, Z. G.; Weaver, M. G.; Wu, K. L.; Pettus, T. R. R. Acc. Chem. Res. 2014, 47, 3655.

[26] Caruana, L.; Fochi, M.; Bernardi, L. Molecules 2015, 20, 11733.

[27] Liang, M.; Zhang, S.; Jia, J.; Tung, C.-H.; Wang, J.; Xu, Z. Org. Lett. 2017, 19, 2526.

[28] Zhao, W.; Wang, Z.; Chu, B.; Sun, J. Angew. Chem., Int. Ed. 2015, $54,1910$.

[29] Huang, Y.; Hayashi, T. J. Am. Chem. Soc. 2015, 137, 7556.

[30] Wang, Z.; Ai, F.; Wang, Z.; Zhao, W.; Zhu, G.; Lin, Z.; Sun, J. J. Am. Chem. Soc. 2015, 137, 383.

[31] Wu, B.; Yu, Z.; Gao, X.; Lan, Y.; Zhou, Y.-G. Angew. Chem., Int. Ed. 2017, 56, 4006.

[32] Chen, P.; Wang, K. 1; Guo, W.; Liu, X.; Liu, Y.; Li, C. Angew. Chem., Int. Ed. 2017, 56, 3689.

[33] Nising, C. F.; Brase, S. Chem. Soc. Rev. 2008, 37, 1218.

[34] Nising, C. F.; Brase, S. Chem. Soc. Rev. 2012, 41, 988.

[35] Heravi, M. M.; Hajiabbasi, P. Mol. Diversity 2014, 18, 411.

[36] Balasubramanian, K.; Selvaraj, S. Synthesis 1977, 767.

[37] Arct, J.; Jakubska, E.; Olszewska, G. Synthesis 1977, 314.

[38] Arct, J.; Jakubska, E.; Olszewska, G. Synth. Commun. 1978, 8, 143.

[39] Samarakoon, T. B.; Hur, M. Y.; Kurtz, R. D.; Hanson, P. R. Org. Lett. 2010, 12, 2182.
[40] Osyanin, V. A.; Nakushnov, V. Y.; Osipov, D. V.; Klimochkin, Y. N. Chem. Heterocycl. Compd. 2010, 46, 1027.

[41] Osyanin, V. A.; Osipov, D. V.; Klimochkin, Y. N. Chem. Heterocycl. Compd. 2010, 46, 377.

[42] Osyanin, V. A.; Nakushnov, V. Y.; Klimochkin, Y. N. Chem. Heterocycl. Compd. 2011, 47, 755 .

[43] Osyanin, V. A.; Klimochkin, Y. N. Chem. Heterocycl. Compd. 2009, $45,833$.

[44] Sidorina, N. E.; Osyanin, V. A. Chem. Heterocycl. Compd. 2005, $41,1201$.

[45] Sidorina, N. E.; Osyanin, V. A. Chem. Heterocycl. Compd. 2007, 43, 1065

[46] Wang, P.; Song, Y.; Zhang, L.; He, H.; Zhou, X. Curr. Med. Chem. $\mathbf{2 0 0 5}, 12,2893$.

[47] Freccero, M. Mini-Rev. Org. Chem. 2004, 1, 403.

[48] Zhang, S.; Peng, D.; Zhao, N.; Yu, Y.; Wang, F.; Liu, H.; Yi, G. Chin. J. Org. Chem. 2019, 39, 555 (in Chinese) (张硕, 彭丹, 赵宁, 于一涛, 王峰, 刘海龙, 伊港, 有机化学, 2019, 39, 555.)

[49] Zhen, M. M.; Zhang, D. F.; Zhang, Z. Y.; Peng, Y. Q. ACS Comb. Sci. 2016, 18, 697.

[50] Erb, W.; Albini, M.; Rouden, J.; Blanchet, J. J. Org. Chem. 2014, $79,10568$.

[51] Katritzky, A. R.; Abdel-Fattah, A. A. A.; Tymoshenko, D. O.; Belyakov, S. A.; Ghiviriga, I.; Steel, P. J. J. Org. Chem. 1999, 64, 6071.

(Zhao, C.) 